\title{
NONZERO POSITIVE SOLUTIONS OF ELLIPTIC SYSTEMS WITH GRADIENT DEPENDENCE AND FUNCTIONAL BCS
}

\author{
STEFANO BIAGI, ALESSANDRO CALAMAI, AND GENNARO INFANTE
}

\begin{abstract}
We discuss, by topological methods, the solvability of systems of second-order elliptic differential equations subject to functional boundary conditions under the presence of gradient terms in the nonlinearities. We prove the existence of non-negative solutions and provide a non-existence result. We present some examples to illustrate the applicability of the existence and non-existence results.
\end{abstract}

\section{INTRODUCTION}

In this paper we study the solvability of a system of second-order elliptic differential equations subject to functional boundary conditions (BCs for short). Namely, we investigate parametric systems of the type

$$
\left\{\begin{array}{lll}
\mathcal{L}_{k} u_{k}=\lambda_{k} f_{k}\left(x, u_{1}, \ldots, u_{m}, \nabla u_{1}, \ldots, \nabla u_{m}\right) & \text { in } \mathcal{O} & (k=1,2, \ldots, m), \\
u_{k}(x)=\eta_{k} \zeta_{k}(x) h_{k}\left[u_{1}, \ldots, u_{m}\right] & \text { for } x \in \partial \mathcal{O} & (k=1,2, \ldots, m),
\end{array}\right.
$$

where $m \geq 1$ is a fixed natural number, $\mathcal{O} \subseteq \mathbb{R}^{n}$ is a bounded and connected open set of class $C^{1, \alpha}$ for some $\alpha \in(0,1)$, and $\lambda_{k}, \eta_{k}, k=1, \ldots, m$, are non-negative real parameters. Moreover $\mathcal{L}_{1}, \ldots, \mathcal{L}_{m}$ are uniformly elliptic, second-order linear partial differential operators (PDOs) in divergence form on $\mathcal{O}$. That is, for $k=1, \ldots, m$,

$$
\mathcal{L}_{k} u:=-\sum_{i, j=1}^{n} \partial_{x_{i}}\left(a_{i, j}^{(k)}(x) \partial_{x_{j}} u+b_{i}^{(k)}(x) u\right)+\sum_{i=1}^{n} c_{i}^{(k)}(x) \partial_{x_{i}} u+d^{(k)}(x) u
$$

where

- the coefficient functions of $\mathcal{L}_{k}$ belong to $C^{1, \alpha}(\overline{\mathcal{O}}, \mathbb{R})$;

- the matrix $A^{(k)}(x):=\left(a_{i, j}^{(k)}(x)\right)_{i, j}$ is symmetric for every $x \in \mathcal{O}$;

- $\mathcal{L}_{k}$ is uniformly elliptic in $\mathcal{O}$, i.e., there exists $\Lambda_{k}>0$ such that

$$
\frac{1}{\Lambda_{k}}\|\xi\|^{2} \leq \sum_{i, j=1}^{n} a_{i, j}^{(k)}(x) \xi_{i} \xi_{j} \leq \Lambda_{k}\|\xi\|^{2} \quad \text { for any } x \in \mathcal{O} \text { and } \xi \in \mathbb{R}^{n} \backslash\{0\}
$$

Date: December 6, 2019.

2010 Mathematics Subject Classification. Primary 35J47, secondary 35B09, 35J57, 35J60, 47H10.

Key words and phrases. Positive solution, elliptic system, gradient terms, functional boundary condition, cone, fixed point index. 
where $\|\xi\|$ stands for the Euclidean norm of $\xi \in \mathbb{R}^{n}$;

- for every non-negative function $\varphi \in C_{0}^{\infty}(\mathcal{O}, \mathbb{R})$ one has

$$
\int_{\mathcal{O}}\left(d^{(k)} \varphi+\sum_{i=1}^{n} b_{i}^{(k)} \partial_{x_{i}} \varphi\right) \mathrm{d} x, \quad \int_{\mathcal{O}}\left(d^{(k)} \varphi+\sum_{i=1}^{n} c_{i}^{(k)} \partial_{x_{i}} \varphi\right) \mathrm{d} x \geq 0 .
$$

Furthermore, for every fixed $k=1, \ldots, m$ we also assume that

- $f_{k}$ is a real-valued continuous function defined on $\overline{\mathcal{O}} \times \mathbb{R}^{m} \times \mathbb{R}^{n m}$;

- $h_{k}$ is a real-valued continuous functional defined on the space $C^{1}\left(\overline{\mathcal{O}}, \mathbb{R}^{m}\right)$;

- $\zeta_{k} \in C^{1, \alpha}(\overline{\mathcal{O}}, \mathbb{R})$ and $\zeta_{k} \geq 0$ on $\mathcal{O}$.

The system (1.1) is quite general, and includes, for example, as a particular case a Dirichlet boundary value problem for elliptic systems with gradient dependence of the form

$$
\begin{cases}-\Delta u_{1}=\lambda_{1} f_{1}\left(x, u_{1}, u_{2}, \nabla u_{1}, \nabla u_{2}\right) & \text { in } \mathcal{O} \\ -\Delta u_{2}=\lambda_{2} f_{2}\left(x, u_{1}, u_{2}, \nabla u_{1}, \nabla u_{2}\right) & \text { in } \mathcal{O} \\ \left.u_{1}\right|_{\partial \mathcal{O}}=0=\left.u_{2}\right|_{\partial \mathcal{O}} & \end{cases}
$$

Systems of nonlinear PDEs of this kind are widely studied in view of applications: in fact, the nonlinearities in (1.2) may depend also on the gradient of the solution, and thus represent convection terms. These problems, in general, are not easily dealt with by means of variational methods. Different approaches in the study of PDEs with gradient terms have been proposed: for example sub- and super-solutions, topological degree theory, mountain pass techniques. We mention, for instance, the pioneering works of Amann and Crandall [3], Brézis and Turner [4, Mawhin and Schmitt [22, 23], Pokhozhaev [25] and the more recent contributions [1, 7, 9, 10, 13, 26, 27, 29]. See also the very recent survey [8] and references therein.

In this paper we adopt a topological approach, based on the classical notion of fixed point index (see e.g. [16]) for the existence result, Theorem 3.3 below, whereas we prove a nonexistence result via an elementary argument. In some sense we follow a path established by Amman [2, 3] and successfully used by many authors in different contexts. We point out that our approach applies not only to Dirichlet BCs but permits to consider (possibly nonlinear) functional BCs, including the special cases of linear (multi-point or integral) BCs of the form

$$
h_{k}[u]=\sum_{j=1}^{m} \sum_{i=1}^{N}\left(\hat{\alpha}_{i j k} u_{j}\left(\omega_{i}\right)+\sum_{l=1}^{n} \hat{\beta}_{i j k l} \partial_{x_{l}} u_{j}\left(\tau_{i}\right)\right)
$$

or

$$
h_{k}[u]=\sum_{j=1}^{m}\left(\int_{\Omega} \hat{\alpha}_{j k}(x) u_{j}(x) d x+\sum_{l=1}^{n} \int_{\Omega} \hat{\beta}_{j k l}(x) \partial_{x_{l}} u_{j}(x) d x\right)
$$


here, in (1.3) $, \hat{\alpha}_{i j k}, \hat{\beta}_{i j k l}$ are non-negative coefficients and $\omega_{i}, \tau_{i} \in \mathcal{O}$ while, in (1.4), $\hat{\alpha}_{j k}, \hat{\beta}_{j k l}$ are non-negative continuous functions on $\overline{\mathcal{O}}$. In particular we observe that nonlinear, nonlocal BCs have seen recently attention in the framework of elliptic equations: we refer the reader to the papers [5, 6, 14, 15, 17, 18, 24] and references therein.

We wish to point out that an advantage of our setting, with respect to the theory developed in [5, 6, 14, 15, 17, 24], is the possibility to allow also gradient dependence within the functionals occurring in the BCs. This follows the approach used recently in [19, 20] within the setting of ODEs.

Note that functional BCs that involve gradient terms may occur in applications. For example, consider a particular case of (1.1) for $m=1$ and $n=2$, namely

$$
\begin{cases}-\Delta u(x)=f(x, u(x), \nabla u(x)), & x \in B \\ u(x)=\eta_{0} u(0)+\eta_{1}\|\nabla u(0)\|, & x \in \partial B\end{cases}
$$

where $B$ is the Euclidean ball in $\mathbb{R}^{2}$ centered at 0 with radius $1,\|\cdot\|$ is the Euclidian norm and $\eta_{i}$ are non-negative coefficients. The BVP (1.5) can be used as a model for the steady states of the temperature of a heated disk of radius 1, where a controller located in the border of the disk adds or removes heat according to the value of the temperature and to its variation, both registered by a sensor located in the center of the disk. In the context of ODEs, a good reference for this kind of thermostat problems is the recent paper [28].

As already pointed out, a peculiarity of system (1.1) is the dependence on the gradient of the solutions, both in the nonlinearity and in the functionals occurring in the BCs, and this represents the main technical difficulty that we have to deal with in this paper. For this purpose, we have to perform a preliminary study of the Green's function of the partial differential operators which occur in (1.1). In Section 2 we collect some properties and estimates on Green's function, which are probably known to the experts in the field, nevertheless we include them for the sake of completeness. Roughly speaking, these estimates yield the a priori bounds needed to compute the fixed point index in suitable cones of non-negative functions.

Section 3 contains our main results, while the final Section 4 includes some examples illustrating our results. In particular, we fix $m=2$ and $n=3$, and, taking into account the parameters $\lambda_{1}, \lambda_{2}, \eta_{1}, \eta_{2}$, we provide existence and non-existence results in some concrete situations.

\section{Preliminaries on DiVERGENCE-Form ELliptiC OPERATORS}

In this Section we present, mostly without proof, several results concerning divergenceform operators which shall play a central rôle in the forthcoming sections. We refer the reader to, e.g., 11, 12 for a detailed treatment of this topic. 
To being with, let $\mathcal{O} \subseteq \mathbb{R}^{n}$ be a fixed open set and let $\mathcal{L}$ be a second-order linear PDO on $\mathcal{O}$ of the following divergence form:

$$
\begin{aligned}
\mathcal{L} u & :=-\sum_{i, j=1}^{n} \partial_{x_{i}}\left(a_{i, j}(x) \partial_{x_{j}} u+b_{i}(x) u\right)+\sum_{i=1}^{n} c_{i}(x) \partial_{x_{i}} u+d(x) u \\
& =-\operatorname{div}(A(x) \nabla u+\mathbf{b} u)+\langle\mathbf{c}, \nabla u\rangle+d u
\end{aligned}
$$

(here, $\mathbf{b}=\left(b_{1}, \ldots, b_{n}\right)$ and $\left.\mathbf{c}=\left(c_{1}, \ldots, c_{n}\right)\right)$. Throughout the sequel, we shall suppose that the following "structural assumptions" on $\mathcal{O}$ and $\mathcal{L}$ are satisfied:

(H0) $\mathcal{O}$ is bounded, connected and of class $C^{1, \alpha}$ for some $\alpha \in(0,1)$;

(H1) the coefficient functions of $\mathcal{L}$ are Hölder-continuous of exponent $\alpha$ up to $\partial \mathcal{O}$, i.e.,

$$
a_{i, j}, b_{i}, c_{i}, d \in C^{\alpha}(\overline{\mathcal{O}}, \mathbb{R}) \quad \text { for every } i, j \in\{1, \ldots, n\}
$$

(H2) the matrix $A(x):=\left(a_{i, j}(x)\right)_{i, j}$ is symmetric in $\mathcal{O}$, i.e.,

$$
a_{i, j}(x)=a_{j, i}(x) \quad \text { for every } x \in \overline{\mathcal{O}} \text { and every } i, j \in\{1, \ldots, n\} \text {; }
$$

(H3) $\mathcal{L}$ is uniformly elliptic in $\mathcal{O}$, i.e., there exists $\Lambda>0$ such that

$$
\frac{1}{\Lambda}\|\xi\|^{2} \leq \sum_{i, j=1}^{n} a_{i, j}(x) \xi_{i} \xi_{j} \leq \Lambda\|\xi\|^{2} \quad \text { for any } x \in \overline{\mathcal{O}} \text { and any } \xi \in \mathbb{R}^{n}
$$

(H4) the inequalities $d-\operatorname{div}(\mathbf{b}) \geq 0$ and $d-\operatorname{div}(\mathbf{c}) \geq 0$ hold in the weak sense of distributions on $\mathcal{O}$, i.e., for every $\varphi \in C_{0}^{\infty}(\mathcal{O}, \mathbb{R})$ such that $\varphi \geq 0$ on $\mathcal{O}$, one has

$$
\int_{\mathcal{O}}\left(d \varphi+\sum_{i=1}^{n} b_{i} \partial_{x_{i}} \varphi\right) \mathrm{d} x \geq 0 \text { and } \int_{\mathcal{O}}\left(d \varphi+\sum_{i=1}^{n} c_{i} \partial_{x_{i}} \varphi\right) \mathrm{d} x \geq 0 .
$$

It should be noticed that, since the coefficient functions of $\mathcal{L}$ are assumed to be just Höldercontinuous on $\overline{\mathcal{O}}$, it is not possible to compute $\mathcal{L} u$ in a point-wise sense (even if $u$ is smooth on $\mathcal{O}$ ); for this reason, the following definition is plainly justified.

Definition 2.1. Let the assumptions (H0)-to-(H4) be in force, and let $f \in L^{2}(\mathcal{O})$. We say that a function $u: \mathcal{O} \rightarrow \mathbb{R}$ is a solution of the equation

$$
\mathcal{L} u=f \quad \text { in } \mathcal{O},
$$

if $u \in W^{1,2}(\mathcal{O})$ and if, for every test function $\phi \in C_{0}^{\infty}(\mathcal{O}, \mathbb{R})$, one has

$$
\int_{\mathcal{O}}(\langle A(x) \nabla u+\mathbf{b} u, \nabla \phi\rangle+\langle\mathbf{c}, \nabla u\rangle \phi+d u \phi) \mathrm{d} x=\int_{\mathcal{O}} f \phi \mathrm{d} x .
$$

Given $g \in W^{1,2}(\mathcal{O})$, we say that $u$ is a solution of the Poisson problem

$$
\left\{\begin{array}{l}
\mathcal{L} u=f \quad \text { in } \mathcal{O}, \\
\left.u\right|_{\partial \mathcal{O}}=g \\
4
\end{array}\right.
$$


if $u$ is a solution of (2.2) and, furthermore, $u-g \in W_{0}^{1,2}(\mathcal{O})$.

Now, as a consequence of the "sign assumption" (H4) it is possible to prove that a suitable form of the Weak Maximum Principle holds for $\mathcal{L}$ (see, e.g., [12, Theorem 8.1]); from this, one can straightforwardly deduce Lemma 2.2 below (see [12, Corollary 8.2]), ensuring that the Poisson problem (2.3) possesses at most one solution.

Lemma 2.2. Let the assumptions $(\mathrm{H} 0)$-to-( $\mathrm{H} 4)$ be in force, and let $u \in W_{0}^{1,2}(\mathcal{O})$ be such that $\mathcal{L} u=0$ or $\mathcal{L}^{T} u=0$ in $\mathcal{O}$. Then $u \equiv 0$ almost everywhere on $\mathcal{O}$.

2.1. The Poisson problem for $\mathcal{L}$. A first group of results we aim to present is about existence and regularity of solutions for the Poisson problem (2.3) for $\mathcal{L}$. In order to do this, we first introduce the following Banach spaces:

- $X=\left(C(\overline{\mathcal{O}}, \mathbb{R}),\|\cdot\|_{\infty}\right)$, where

$$
\|f\|_{\infty}:=\max _{x \in \overline{\mathcal{O}}}|f(x)|
$$

- $X=\left(C^{1}(\overline{\mathcal{O}}, \mathbb{R}),\|\cdot\|_{C^{1}(\overline{\mathcal{O}}, \mathbb{R})}\right)$, where

$$
\|f\|_{C^{1}(\overline{\mathcal{O}}, \mathbb{R})}:=\max _{j=1, \ldots, n}\left\{\|f\|_{\infty},\left\|\partial_{j} f\right\|_{\infty}: j=1, \ldots, n\right\}
$$

- $X=C^{1, \theta}(\overline{\mathcal{O}}, \mathbb{R})$ (for some $\left.\theta \in(0,1)\right)$, where

$$
\|u\|_{C^{1, \theta}(\overline{\mathcal{O}}, \mathbb{R})}:=\max _{j=1, \ldots, n}\left\{\|u\|_{\infty},\left\|\partial_{j} u\right\|_{\infty}, \sup _{x, y \in \overline{\mathcal{O}}} \frac{\left|\partial_{j} u(x)-\partial_{j} u(y)\right|}{\|x-y\|^{\theta}}\right\}
$$

Given $f \in C^{1}(\overline{\mathcal{O}}, \mathbb{R})$, it will be also convenient to define, with abuse of notation,

$$
\|\nabla f\|_{\infty}:=\max _{j=1, \ldots, n}\left\{\left\|\partial_{j} f\right\|_{\infty}: j=1, \ldots, n\right\}
$$

so that, clearly, $\|f\|_{C^{1}(\overline{\mathcal{O}}, \mathbb{R})}=\max \left\{\|f\|_{\infty},\|\nabla f\|_{\infty}\right\}$.

Now, by exploiting assumptions (H3)-(H4), Lemma 2.2 and the Fredholm alternative, one can establish the following basic theorem (for a proof, see [12, Theorem 8.3]).

Theorem 2.3. Let the assumptions $(\mathrm{H} 0)$-to-(H4) be in force. Then, for every $f \in L^{2}(\mathcal{O})$ and every $g \in W^{1,2}(\mathcal{O})$ there exists a unique solution $u_{f, g} \in W^{1,2}(\mathcal{O})$ of $(2.3)$.

Throughout the sequel, we indicate by $u_{f, g}$ the unique solution in $W^{1,2}(\mathcal{O})$ of (2.3) (for fixed $f \in L^{2}(\mathcal{O})$ and $g \in W^{1,2}(\mathcal{O})$ ), whose existence is guaranteed by Theorem 2.3. In the particular case when $g \equiv 0$, we simply write $u_{f}$ instead of $u_{f, 0}$.

Remark 2.4. Theorem 2.3 holds under more general hypotheses: in fact, it suffices to assume that $\mathcal{O}$ is bounded and that the coefficient functions of $\mathcal{L}$ are in $L^{\infty}(\mathcal{O})$. 
Remark 2.5. Let $f_{1}, f_{2} \in L^{2}(\mathcal{O})$ and, for $i=1,2$, let $u_{i}=u_{f_{i}} \in W_{0}^{1,2}(\mathcal{O})$ be the unique solution of (2.3) with $f=f_{i}$ (and $g \equiv 0$ ). Since, obviously, it holds that

$$
\mathcal{L}\left(u_{f_{1}}+u_{f_{2}}\right)=u_{f_{1}}+u_{f_{2}} \quad \text { and } \quad u_{f_{1}}+u_{f_{2}} \in W_{0}^{1,2}(\mathcal{O}),
$$

we conclude that the unique solution of (2.3) with $f=f_{1}+f_{2}$ and $g \equiv 0$ is $u_{f_{1}}+u_{f_{2}}$.

Since we aim to apply suitable fixed-point techniques to operators acting on spaces of $C^{1}$ functions, we are interested in solving (2.3) for continuous $f$ and regular $g$. In this context, the unique solution $u_{f, g}$ of (2.3) turns out to be much more regular that $W^{1,2}$; in fact, we have the following crucial result (for a proof, see [12, Thm.s 8.16, 8.33 and 8.34]).

Theorem 2.6. Let the assumptions (H0)-to-(H4), and let $\mathcal{L}$ be as in (2.1). Moreover, let $f \in C(\overline{\mathcal{O}}, \mathbb{R})$ and let $g \in C^{1, \alpha}(\overline{\mathcal{O}}, \mathbb{R})$. Then the following facts hold true.

(i) There exists a unique $\hat{u}_{f, g} \in C^{1, \alpha}(\overline{\mathcal{O}}, \mathbb{R})$ such that

$$
\hat{u}_{f, g} \equiv u_{f, g} \text { a.e. on } \mathcal{O} \text {. }
$$

In particular, $\hat{u}_{f, g}$ solves (2.2) and $\hat{u}_{f, g} \equiv g$ point-wise on $\partial \Omega$.

(ii) There exists a constant $C>0$, only depending on $n, \Lambda$ and $\mathcal{O}$, such that

$$
\left\|\hat{u}_{f, g}\right\|_{C^{1, \alpha}(\overline{\mathcal{O},}, \mathbb{R})} \leq C\left(\|f\|_{C(\overline{\mathcal{O}}, \mathbb{R})}+\|g\|_{C^{1, \alpha}(\overline{\mathcal{O},}, \mathbb{R})}\right) .
$$

(iii) If $f \geq 0$ on $\overline{\mathcal{O}}$ and $g \geq 0$ on $\partial \mathcal{O}$, then $\hat{u}_{f, g} \geq 0$ on $\overline{\mathcal{O}}$.

Now, in view of Theorem 2.6-(i), we can define a linear operator as follows

$$
\mathcal{G}_{\mathcal{L}}: C(\overline{\mathcal{O}}, \mathbb{R}) \longrightarrow C^{1, \alpha}(\overline{\mathcal{O}}, \mathbb{R}), \quad \mathcal{G}_{\mathcal{L}}(f):=\hat{u}_{f},
$$

where $\hat{u}_{f}=\hat{u}_{f, 0} \in C^{1, \alpha}(\overline{\mathcal{O}}, \mathbb{R})$ is the unique solution of (2.3) with $g \equiv 0$. We shall call $\mathcal{G}_{\mathcal{L}}$ the Green operator for $\mathcal{L}$. By exploiting assertions (ii)-(iii) of Theorem 2.6, it is possible to deduce some continuous-compactness properties of $\mathcal{G}_{\mathcal{L}}$ which shall play a central rôle in the next sections; to be more precise, we have the following proposition.

Proposition 2.7. Let the assumptions $(\mathrm{H} 0)$-to-(H4) be in force, and let $\mathcal{G}_{\mathcal{L}}$ be the operator defined in (2.9). Then the following facts hold:

(i) $\mathcal{G}_{\mathcal{L}}$ is continuous from $C(\overline{\mathcal{O}}, \mathbb{R})$ to $C^{1, \alpha}(\overline{\mathcal{O}}, \mathbb{R})$;

(ii) $\mathcal{G}_{\mathcal{L}}$ is compact from $C(\overline{\mathcal{O}}, \mathbb{R})$ to $C^{1}(\overline{\mathcal{O}}, \mathbb{R}) \supseteq C^{1, \alpha}(\overline{\mathcal{O}}, \mathbb{R})$;

(iii) if $V_{0}:=C\left(\overline{\mathcal{O}}, \mathbb{R}^{+}\right) \subseteq C(\overline{\mathcal{O}}, \mathbb{R})$ denotes the (convex) cone of the non-negative continuous functions on $\overline{\mathcal{O}}$, it holds that $\mathcal{G}_{\mathcal{L}}\left(V_{0}\right) \subseteq V_{0}$.

Proof. (i) On account of Theorem 2.64(ii), for every $f \in C(\overline{\mathcal{O}}, \mathbb{R})$ one has

$$
\left\|\mathcal{G}_{\mathcal{L}}(f)\right\|_{C^{1, \alpha}(\overline{\mathcal{O}}, \mathbb{R})}^{6} \leq C\|f\|_{\infty}
$$


(here, $C>0$ is a constant independent of $f$ ). Since $\mathcal{G}_{\mathcal{L}}$ is linear (see Remark 2.5), from (2.10) we immediately deduce that $\mathcal{G}_{\mathcal{L}}$ is continuous from $C(\overline{\mathcal{O}}, \mathbb{R})$ to $C^{1, \alpha}(\overline{\mathcal{O}}, \mathbb{R})$.

(ii) Let $\left\{f_{j}\right\}_{j}$ be a bounded sequence in $C(\overline{\mathcal{O}}, \mathbb{R})$. On account of $(2.10)$, we see that the sequence $\left\{\mathcal{G}_{\mathcal{L}}\left(f_{j}\right)\right\}_{j}$ is bounded in $C^{1, \alpha}(\overline{\mathcal{O}}, \mathbb{R})$; as a consequence, a standard application of Arzel-Ascoli's Theorem implies the existence of $u_{0}, \ldots, u_{n} \in C(\overline{\mathcal{O}}, \mathbb{R})$ such that

(a) $\left\|\mathcal{G}_{\mathcal{L}}\left(f_{j_{k}}\right)-u_{0}\right\|_{\infty} \rightarrow 0$ as $k \rightarrow \infty$,

(b) $\left\|\partial_{i}\left(\mathcal{G}_{\mathcal{L}}\left(f_{j_{k}}\right)\right)-u_{i}\right\|_{\infty} \rightarrow 0$ as $k \rightarrow \infty$ (for every $\left.i=1, \ldots, n\right)$,

where $\left\{f_{j_{k}}\right\}_{k}$ is a suitable sub-sequence of $\left\{f_{j}\right\}_{j}$. By combining (a) and (b), we deduce that $u_{0} \in C^{1}(\overline{\mathcal{O}}, \mathbb{R})$ and that $\nabla u_{0}=\left(u_{1}, \ldots, u_{n}\right)$; moreover, one has

$$
\left\|\mathcal{G}_{\mathcal{L}}\left(f_{j_{k}}\right)-u_{0}\right\|_{\left.C^{1}(\overline{\mathcal{O}}), \mathbb{R}\right)} \rightarrow 0 \quad \text { as } k \rightarrow \infty
$$

and this proves that $\mathcal{G}_{\mathcal{L}}$ is compact from $C(\overline{\mathcal{O}}, \mathbb{R})$ to $C^{1}(\overline{\mathcal{O}}, \mathbb{R})$, as desired.

(iii) Let $f \in V_{0}$ be fixed. Since, by Theorem 2.6 (iii), we know that $\mathcal{G}_{\mathcal{L}}(f)=\hat{u}_{f, 0} \geq 0$ throughout $\overline{\mathcal{O}}$, we immediately conclude that $\mathcal{G}_{\mathcal{L}}(f) \in V_{0} \cap C^{1, \alpha}(\overline{\mathcal{O}}, \mathbb{R})$, as desired.

2.2. Green's function for $\mathcal{L}$. Now we have established Proposition 2.7, we turn to present a second group of results: this is about the existence of a Green's function for $\mathcal{L}$ allowing to obtain an integral representation formula for $\mathcal{G}_{\mathcal{L}}$.

To begin with, we demonstrate the following key theorem.

Theorem 2.8. Let the assumptions (H0)-to-(H4) be in force, and let $\mathcal{L}$ be as in (2.1). There exists a function $g_{\mathcal{L}}: \mathcal{O} \times \mathcal{O} \rightarrow[0, \infty)$ such that

(a) $g_{\mathcal{L}}(\cdot ; x) \in L^{1}(\mathcal{O})$ for almost every $x \in \mathcal{O}$;

(b) for every $f \in C(\overline{\mathcal{O}}, \mathbb{R})$ one has

$$
\mathcal{G}_{\mathcal{L}}(f)(x)=\int_{\mathcal{O}} g_{\mathcal{L}}(y ; x) f(y) \mathrm{d} y \quad \text { for a.e. } x \in \mathcal{O} .
$$

Furthermore, $g_{\mathcal{L}}$ enjoys the following properties:

(I) there exists a constant $c_{0}>0$ such that, for a.e. $x, y \in \mathcal{O}$, one has

$$
0 \leq g_{\mathcal{L}}(y ; x) \leq c_{0}\|x-y\|^{2-n}
$$

(II) $g_{\mathcal{L}}(\cdot ; x) \in W_{0}^{1, p}(\mathcal{O})$ for a.e. $x \in \mathcal{O}$ and every $1 \leq p<n /(n-1)$;

(III) $g_{\mathcal{L}}(y ; \cdot) \in W_{0}^{1, p}(\mathcal{O})$ for a.e. $y \in \mathcal{O}$ and every $1 \leq p<n /(n-1)$;

$(I V)$ there exists a constant $c_{1}>0$ such that, for a.e. $x, y \in \mathcal{O}$, one has

$$
\left\|\nabla_{y} g_{\mathcal{L}}(y ; x)\right\| \leq c_{1}\|x-y\|^{1-n} \quad \text { and } \quad\left\|\nabla_{x} g_{\mathcal{L}}(y ; x)\right\| \leq c_{1}\|x-y\|^{1-n} .
$$


Finally, $g_{\mathcal{L}}$ is unique in the following sense: if $\tilde{g}: \mathcal{O} \times \mathcal{O} \rightarrow[0, \infty)$ is another function satisfying $(\mathrm{a})-(\mathrm{b})$, then $g_{\mathcal{L}}(\cdot ; x)=\tilde{g}(\cdot ; x)$ in $L^{1}(\mathcal{O})$ for a.e. $x \in \mathcal{O}$.

Throughout the sequel, we shall refer to the function $g_{\mathcal{L}}$ in Theorem 2.8 as the Green's function for the operator $\mathcal{G}_{\mathcal{L}}$ (and related to the open set $\mathcal{O}$ ).

Proof. We begin by proving the existence part of the theorem. In order to do this, we make pivotal use of several results established in the very recent paper [21].

First of all, by [21, Proposition 5.3] there exists a function $g_{\mathcal{L}}: \mathcal{O} \times \mathcal{O} \rightarrow \mathbb{R}$ such that

(i) $g_{\mathcal{L}}(\cdot ; x) \in W^{1, p}(\mathcal{O})$ for a.e. $x \in \mathcal{O}$ and every $1 \leq p<n /(n-1)$;

(ii) for every fixed $f \in C(\overline{\mathcal{O}}, \mathbb{R})$ one has

$$
\mathcal{G}_{\mathcal{L}}(f)(x)=\int_{\mathcal{O}} g_{\mathcal{L}}(y ; x) f(y) \mathrm{d} y \quad \text { for a.e. } x \in \mathcal{O} .
$$

Moreover, by [21, Theorem 6.10] we also have that

$$
0 \leq g_{\mathcal{L}}(y ; x) \leq c_{0}\|x-y\|^{2-n} \quad \text { for a.e. } x, y \in \mathcal{O} \text { with } x \neq y,
$$

where $c_{0}>0$ is a suitable constant. In view of these facts, to complete the demonstration we are left to prove assertion (iii) and the point-wise estimates in (2.13).

To this end, let us introduce the so-called (formal) adjoint $\mathcal{L}^{T}$ of $\mathcal{L}$ : this is the linear differential operator defined on $\mathcal{O}$ in the following way

$$
\begin{aligned}
\mathcal{L}^{T} v & :=-\sum_{i, j=1}^{n} \partial_{x_{i}}\left(a_{i, j}(x) \partial_{x_{j}} v+c_{i}(x) v\right)+\sum_{i=1}^{n} b_{i}(x) \partial_{x_{i}} v+d(x) v \\
& =-\operatorname{div}(A(x) \nabla v+\mathbf{c} v)+\langle\mathbf{b}, \nabla v\rangle+d v .
\end{aligned}
$$

Clearly, $\mathcal{L}^{T}$ takes the same divergence-form of $\mathcal{L}$ in (2.1) (with $\mathbf{b}$ and $\mathbf{c}$ interchanged); furthermore, due to the "symmetry" in assumption (H4), it is readily seen that $\mathcal{L}^{T}$ satisfies the "structural assumptions" (H1)-to-(H4).

As a consequence, all the results established so far do apply to $\mathcal{L}^{T}$. In particular, for every fixed $g \in C(\overline{\mathcal{O}}, \mathbb{R})$ there exists a unique function $\mathcal{T}(g) \in C^{1, \alpha}(\overline{\mathcal{O}}, \mathbb{R})$ such that

$$
\mathcal{L}^{T} \mathcal{T}(g)=g \quad \text { in } \mathcal{O} \quad \text { and } \quad \mathcal{T}(g) \equiv 0 \quad \text { on } \partial \mathcal{O} .
$$

Now, by [21, Theorem 6.12] there exists a function $G: \mathcal{O} \times \mathcal{O} \rightarrow \mathbb{R}$ such that

(iii) $G(\cdot ; y) \in W^{1, p}(\mathcal{O})$ for a.e. $y \in \mathcal{O}$ and every $1 \leq p<n /(n-1)$;

(iv) for every fixed $g \in C(\overline{\mathcal{O}}, \mathbb{R})$ one has

$$
\mathcal{T}(g)(y)=\int_{\mathcal{O}} G(x ; y) g(x) \mathrm{d} x \quad \text { for a.e. } y \in \mathcal{O} .
$$


On the other hand, since [21, Proposition 6.13] shows that

$$
G(x ; y)=g_{\mathcal{L}}(y ; x) \quad \text { for a.e. } x, y \in \mathcal{O} \text { with } x \neq y,
$$

from (iii) we infer that $g_{\mathcal{L}}(y ; \cdot)=G(\cdot ; y) \in W^{1, p}(\mathcal{O})$ for almost every $y \in \mathcal{O}$ and every exponent $p \in[1, n /(n-1))$. This is exactly assertion (III).

Finally, we prove the point-wise estimates in assertion (IV). First of all, since $\mathcal{L}$ satisfies assumptions (H1)-to-(H4), we are entitled to apply [21, Theorem 8.1], ensuring that

$$
\left\|\nabla_{x} G(x ; y)\right\| \leq c_{1}^{\prime}\|x-y\|^{1-n} \quad \text { for a.e. } x, y \in \mathcal{O} \text { with } x \neq y,
$$

where $c_{1}^{\prime}>0$ is a suitable constant. Moreover, since also $\mathcal{L}^{T}$ satisfies assumptions (H1)-to(H4), another application of [21, Theorem 8.1] gives

$$
\left\|\nabla_{y} g_{\mathcal{L}}(y ; x)\right\| \leq c_{1}^{\prime \prime}\|x-y\|^{1-n} \quad \text { for a.e. } x, y \in \mathcal{O} \text { with } x \neq y,
$$

where $c_{1}^{\prime \prime}>0$ is another suitable constant. Gathering together (2.17), (2.16) and (2.15) we immediately obtain the desired (2.13) (with $\left.c_{1}:=\max \left\{c_{1}^{\prime}, c_{1}^{\prime \prime}\right\}\right)$.

As for the uniqueness part of the theorem, let us suppose that there exists another function $\tilde{g}: \mathcal{O} \times \mathcal{O} \rightarrow[0, \infty)$ satisfying (a)-(b). In particular, for every $\phi \in C_{0}^{\infty}(\mathcal{O}, \mathbb{R})$ one has

$$
\int_{\mathcal{O}}\left(g_{\mathcal{L}}(y ; x)-\tilde{g}(y ; x)\right) \phi(y) \mathrm{d} y=0 \quad \text { for a.e. } x \in \mathcal{O} .
$$

Now, the space $C_{0}^{\infty}(\mathcal{O}, \mathbb{R})$ being separable (with its usual LF-topology), there exists a countable set $\mathcal{F} \subseteq C_{0}^{\infty}(\mathcal{O}, \mathbb{R})$ which is dense; moreover, by (2.18), for every $\phi \in \mathcal{F}$ there exists a set $E(\phi) \subseteq \mathcal{O}$, with zero-Lebesgue measure, such that

$$
\int_{\mathcal{O}}\left(g_{\mathcal{L}}(y ; x)-\tilde{g}(y ; x)\right) \phi(y) \mathrm{d} y=0 \quad \text { for all } x \in E(\phi) .
$$

We then define $E:=\cup_{\phi \in \mathcal{F}} E(\phi)$. Since $\mathcal{F}$ is countable and $E(\phi)$ has zero-Lebesgue measure for every $\phi$, we see that $E$ has measure zero; moreover, for every $x \in \mathcal{O} \backslash E$ we have

$$
\int_{\mathcal{O}}\left(g_{\mathcal{L}}(y ; x)-\tilde{g}(y ; x)\right) \phi(y) \mathrm{d} y=0 \quad \text { for all } \phi \in \mathcal{F} .
$$

This proves that, for every $x \in \mathcal{O} \backslash E$, the distribution $g_{\mathcal{L}}(\cdot ; x)-\tilde{g}(\cdot ; x)$ vanishes on $\mathcal{F}$; the latter being dense, we then conclude that $g_{\mathcal{L}}(\cdot ; x)=\tilde{g}(\cdot ; x)$ in $L^{1}(\mathcal{O})$ for a.e. $x, y \in \mathcal{O}$.

This ends the proof.

Remark 2.9. The approach adopted for the proof of Theorem 2.8 shows the reason why we have assumed that $d-\operatorname{div}(\mathbf{b}) \geq 0$ and $d-\operatorname{div}(\mathbf{c}) \geq 0$ in the sense of distributions.

In fact, under this assumption, all the mentioned results in [21] hold both for $\mathcal{L}$ and for its transpose $\mathcal{L}^{T}$; in particular, this allows us to obtain point-wise estimates both for

$$
\nabla_{x} g_{\mathcal{L}}(y ; x)=\nabla_{x} G(x ; y)_{9} \quad \text { and } \quad \nabla_{y} g_{\mathcal{L}}(y ; x)
$$


Remark 2.10. It is contained in the proof of Theorem 2.8 the following fact: if $\mathcal{L}$ is of the form (2.1) and if $\mathbf{b} \equiv \mathbf{c}$ on $\mathcal{O}$, then the Green's function for $\mathcal{G}_{\mathcal{L}}$ is symmetric, that is,

$$
g_{\mathcal{L}}(y ; x)=g_{\mathcal{L}}(x ; y) \quad \text { for a.e. } x, y \in \mathcal{O} \text {. }
$$

In fact, if $\mathbf{b} \equiv \mathbf{c}$ on $\mathcal{O}$, then the adjoint operator $\mathcal{L}^{T}$ coincides with $\mathcal{L}$ (see (2.14) $)$; thus, following the notation in the proof of Theorem 2.8, we have

$$
g_{\mathcal{L}}(x ; y)=G(x ; y)=g_{\mathcal{L}}(y ; x) .
$$

Remark 2.11. By carefully scrutinizing the proofs of the existence results for $g_{\mathcal{L}}$ contained in [21, Proposition 5.3], one can recognize that the following properties hold:

(a) for a.e. $x \in \mathcal{O}$ and every $\epsilon>0$, we have $g_{\mathcal{L}}(\cdot ; x) \in W^{1,2}(\mathcal{O} \backslash B(x, \epsilon))$;

(b) $g_{\mathcal{L}}(\cdot ; x)$ is a solution of $\mathcal{L}^{T} u=0$ in $\mathcal{O} \backslash B(x, \epsilon)$, where $\mathcal{L}^{T}$ is as in (2.14).

Analogously, an inspection to the proof of [21, Theorem 6.12] shows that

(a') for a.e. $y \in \mathcal{O}$ and every $\epsilon>0$, we have $G(\cdot ; y)=g_{\mathcal{L}}(y ; \cdot) \in W^{1,2}(\mathcal{O} \backslash B(y, \epsilon))$;

(b') $G(\cdot ; y)=g_{\mathcal{L}}(y ; \cdot)$ is a solution of $\mathcal{L} u=0$ in $\mathcal{O} \backslash B(y, \epsilon)$.

Gathering together all these facts, from the classical elliptic regularity theory (see, e.g., [12, Corollary 8.36]) we deduce that $g_{\mathcal{L}}$ is of class $C^{1, \alpha}$ out of the diagonal of $\mathcal{O} \times \mathcal{O}$.

We now use the point-wise estimates in (2.12)-(2.13) to prove the following lemma.

Lemma 2.12. Let the assumptions $(\mathrm{H} 0)$-to-(H4) be in force, and let $g_{\mathcal{L}}$ be the Green's function for $\mathcal{G}_{\mathcal{L}}$. Moreover, let $\rho:=\operatorname{diam}(\mathcal{O})$. Then, the following estimates hold:

$$
\begin{aligned}
& \int_{\mathcal{O}} g_{\mathcal{L}}(y ; x) \mathrm{d} y \leq c_{0} \cdot \frac{n \omega_{n} \rho^{2}}{2} \quad \text { for a.e. } x \in \mathcal{O} ; \\
& \int_{\mathcal{O}}\left|\partial_{x_{i}} g_{\mathcal{L}}(y ; x)\right| \mathrm{d} y \leq c_{1} \cdot n \omega_{n} \rho . \quad \text { for a.e. } x \in \mathcal{O} .
\end{aligned}
$$

Here, $\omega_{n}$ is the Lebesgue measure of the unit ball $B(0,1) \subseteq \mathbb{R}^{n}$.

Proof. We begin by proving (2.19). To this end we first notice that, if $x \in \mathcal{O}$ is arbitrary, then $\mathcal{O} \subseteq \overline{B(x, \rho)}$; as a consequence, by crucially exploiting estimate $(\underline{2.12})$ we get

$$
\begin{aligned}
\int_{\mathcal{O}} g_{\mathcal{L}}(y ; x) \mathrm{d} y & \leq c_{0} \int_{\mathcal{O}}\|x-y\|^{2-n} \mathrm{~d} y \leq c_{0} \int_{\overline{B(x, \rho)}}\|x-y\|^{2-n} \mathrm{~d} y \\
& =c_{0} \int_{\overline{B(0, \rho)}}\|y\|^{2-n} \mathrm{~d} y=c_{0} \int_{0}^{\rho} t^{2-n} \mathcal{H}^{n-1}(\partial B(0, t)) \mathrm{d} t \\
& =c_{0} n \omega_{n} \int_{0}^{\rho} t \mathrm{~d} t=c_{0} \cdot \frac{n \omega_{n} \rho^{2}}{2}
\end{aligned}
$$


which is exactly the desired (2.19). As for the proof of (2.20), we argue essentially in the same way: by crucially exploiting the estimate (2.13) we get

$$
\begin{aligned}
\int_{\mathcal{O}}\left|\partial_{x_{i}} g_{\mathcal{L}}(y ; x)\right| \mathrm{d} y & \leq c_{1} \int_{\mathcal{O}}\|x-y\|^{1-n} \mathrm{~d} y \leq c_{1} \int_{\overline{B(x, \rho)}}\|x-y\|^{1-n} \mathrm{~d} y \\
& =c_{1} \int_{\overline{B(0, \rho)}}\|y\|^{1-n} \mathrm{~d} y=c_{1} \int_{0}^{\rho} t^{1-n} \mathcal{H}^{n-1}(\partial B(0, t)) \mathrm{d} t \\
& =c_{1} n \omega_{n} \int_{0}^{\rho} \mathrm{d} t=c_{1} \cdot n \omega_{n} \rho,
\end{aligned}
$$

and this is precisely the desired inequality (2.20).

Remark 2.13. We explicitly observe that, by combining the estimate (2.19) in Lemma 2.12 with the representation formula (2.11), for a.e. $x \in \mathcal{O}$ we obtain

$$
0 \leq \mathcal{G}_{\mathcal{L}}(\hat{1})(x)=\int_{\mathcal{O}} g(y ; x) \mathrm{d} y \leq c_{0} \cdot \frac{n \omega_{n} \rho^{2}}{2},
$$

where $\rho:=\operatorname{diam}(\mathcal{O})$ and $\hat{1}$ denotes the constant function equal to 1 on $\mathcal{O}$. As a consequence, since $\mathcal{G}_{\mathcal{L}}(\hat{1}) \in C(\overline{\mathcal{O}}, \mathbb{R})$, we get

$$
\left\|\mathcal{G}_{\mathcal{L}}(\hat{1})\right\|_{\infty} \leq c_{0} \cdot \frac{n \omega_{n} \rho^{2}}{2} .
$$

We conclude this part of the Section by deducing from (2.11) an integral representation for the $x_{i}$-derivatives of $\mathcal{G}_{\mathcal{L}}(f)$. To this end we first observe that, if $f \in C(\overline{\mathcal{O}}, \mathbb{R})$, Lemma 2.12 ensures that the following "potential-type" functions are well-defined:

$$
\mathcal{P}^{(i)} f(x):=\int_{\mathcal{O}} \partial_{x_{i}} g_{\mathcal{L}}(y ; x) f(y) \mathrm{d} y \quad(\text { for } i=1, \ldots, n) .
$$

In fact, by estimate (2.20) in Lemma 2.12 we have (for $i=1, \ldots, n$ )

$$
\begin{gathered}
\int_{O}\left|\partial_{x_{i}} g_{\mathcal{L}}(y ; x)\right| \cdot|f(y)| \mathrm{d} y \leq\|f\|_{\infty} \cdot \int_{\mathcal{O}}\left|\partial_{x_{i}} g_{\mathcal{L}}(y ; x)\right| \mathrm{d} y \\
\leq\|f\|_{\infty} \cdot c_{1} n \omega_{n} \operatorname{diam}(\mathcal{O}) \quad(\text { for a.e. } x \in \mathcal{O}) .
\end{gathered}
$$

Moreover, from the above computation we also infer that (again for $i=1, \ldots, n$ )

$$
\mathcal{P}^{(i)} f \in L^{\infty}(\mathcal{O}) \text { and }\left\|\mathcal{P}^{(i)} f\right\|_{L^{\infty}(\mathcal{O})} \leq\|f\|_{\infty} \cdot c_{1} n \omega_{n} \operatorname{diam}(\mathcal{O}) .
$$

We are then ready to prove the following Proposition.

Proposition 2.14. Let the assumptions ( $\mathrm{H} 0)$-to- $(\mathrm{H} 4)$ be in force, and let $f \in C(\overline{\mathcal{O}}, \mathbb{R})$. Moreover, let $i \in\{1, \ldots, n\}$ be fixed, and let $\mathcal{P}^{(i)} f$ be as in (2.21). Then, we have

$$
\partial_{x_{i}} \mathcal{G}_{\mathcal{L}}(f)(x)=\mathcal{P}^{(i)} f(x)=\int_{\mathcal{O}} \partial_{x_{i}} g_{\mathcal{L}}(y ; x) f(y) \mathrm{d} y \quad \text { for a.e. } x \in \mathcal{O} .
$$


Proof. We first notice, since $\mathcal{G}_{\mathcal{L}}(f) \in C^{1, \alpha}(\overline{\mathcal{O}}, \mathbb{R})$, the identity $(2.22)$ follows if we show that the $L^{\infty}$-function $\mathcal{P}^{(i)} f$ is the weak derivative (in $L^{1}(\mathcal{O})$ ) of $\mathcal{G}_{\mathcal{L}}(f)$. To prove this fact, we argue as follows: firstly, if $\phi \in C_{0}^{\infty}(\mathcal{O}, \mathbb{R})$, by the estimate (2.19) in Lemma 2.12 we get

$$
\begin{aligned}
\int_{\mathcal{O} \times \mathcal{O}} g_{\mathcal{L}}(y ; x) \cdot|f(y)| \cdot\left|\partial_{x_{i}} \phi(x)\right| \mathrm{d} x \mathrm{~d} y \\
\quad \leq\|f\|_{C(\overline{\mathcal{O}}, \mathbb{R})} \cdot\left\|\partial_{i} \phi\right\|_{\infty} \cdot \int_{\mathcal{O}}\left(\int_{\mathcal{O}} g_{\mathcal{L}}(y ; x) \mathrm{d} y\right) \mathrm{d} x \\
\quad \leq\|f\|_{C(\overline{\mathcal{O}}, \mathbb{R})} \cdot\left\|\partial_{i} \phi\right\|_{\infty} \cdot c_{0} \cdot \frac{n \omega_{n} \operatorname{diam}(\mathcal{O})^{2}}{2} \cdot|\mathcal{O}|
\end{aligned}
$$

we are then entitled to apply Fubini's Theorem, obtaining

$$
\begin{gathered}
\int_{\mathcal{O}} \mathcal{G}_{\mathcal{L}}(f)(x) \partial_{x_{i}} \phi(x) \mathrm{d} x=\int_{\mathcal{O}}\left(\int_{\mathcal{O}} g_{\mathcal{L}}(y ; x) f(y) \mathrm{d} y\right) \partial_{x_{i}} \phi(x) \mathrm{d} x \\
=\int_{\mathcal{O}}\left(\int_{\mathcal{O}} g_{\mathcal{L}}(y ; x) \partial_{x_{i}} \phi(x) \mathrm{d} x\right) f(y) \mathrm{d} y \\
\left.\quad \text { since } g_{\mathcal{L}}(y ; \cdot) \in W_{0}^{1,1}(\mathcal{O}), \text { see Theorem 2.8-(III) }\right) \\
=-\int_{\mathcal{O}}\left(\int_{\mathcal{O}} \partial_{x_{i}} g_{\mathcal{L}}(y ; x) \phi(x) \mathrm{d} x\right) f(y) \mathrm{d} y=:(\star) .
\end{gathered}
$$

On the other hand, since the estimate (2.20) in Lemma 2.12 implies that

$$
\begin{aligned}
\int_{\mathcal{O} \times \mathcal{O}} \mid & \partial_{x_{i}} g_{\mathcal{L}}(y ; x)|\cdot| f(y)|\cdot| \phi(x) \mid \mathrm{d} x \mathrm{~d} y \\
& \leq\|f\|_{\infty} \cdot\|\phi\|_{\infty} \cdot \int_{\mathcal{O}}\left(\int_{\mathcal{O}}\left|\partial_{x_{i}} g_{\mathcal{L}}(y ; x)\right| \mathrm{d} y\right) \mathrm{d} x \\
& \leq\|f\|_{\infty} \cdot\|\phi\|_{\infty} \cdot c_{1} \cdot n \omega_{n} \operatorname{diam}(\mathcal{O}) \cdot|\mathcal{O}|
\end{aligned}
$$

another application of Fubini's Theorem is legitimate, and we get

$$
(\star)=-\int_{\mathcal{O}}\left(\int_{\mathcal{O}} \partial_{x_{i}} g_{\mathcal{L}}(y ; x) f(y) \mathrm{d} y\right) \phi(x) \mathrm{d} x \stackrel{\sqrt{2.21}}{=}-\int_{\mathcal{O}} \mathcal{P}^{(i)} f(x) \phi(x) \mathrm{d} x .
$$

Due to the arbitrariness of $\phi \in C_{0}^{\infty}(\overline{\mathcal{O}}, \mathbb{R})$, we then conclude that $\mathcal{P}^{(i)} f$ is the weak derivative of $\mathcal{G}_{\mathcal{L}}(f)$ in $L^{1}(\mathcal{O})$, and the proof is complete.

Remark 2.15. By using the regularity of $g_{\mathcal{L}}$ described in Remark 2.11, it is quite standard to recognize that, for a fixed $f \in C(\overline{\mathcal{O}}, \mathbb{R})$, the functions

$$
\mathcal{O} \ni x \mapsto \int_{\mathcal{O}} g_{\mathcal{L}}(y ; x) f(y) \mathrm{d} y \quad \text { and } \quad \mathcal{P}^{(1)} f, \ldots, \mathcal{P}^{(n)} f
$$

are continuous on $\mathcal{O}$. As a consequence, the representation formulas (2.11) and (2.22) actually hold true for every $x \in \mathcal{O}$ (not only almost everywhere). 
2.3. Spectral properties of $\mathcal{G}_{\mathcal{L}}$. We conclude this section by briefly turning our attention to the spectral properties of the Green's operator $\mathcal{G}_{\mathcal{L}}$.

To begin with, we remind the following theorem (see, e.g., [12, Theorem 8.6]).

Theorem 2.16. Let the assumptions (H0)-to-(H4) be in force. Then, there exists a countable and discrete set $\Sigma \subseteq(0, \infty)$ with the following property: for every $\sigma \in \Sigma$ the subspace of solutions of the homogeneous problem

$$
\left\{\begin{array}{l}
\mathcal{L} u=\sigma u \quad \text { in } \mathcal{O}, \\
\left.u\right|_{\partial \mathcal{O}}=0
\end{array}\right.
$$

has positive finite dimension (as a subspace of $W^{1,2}(\mathcal{O})$ ).

By making use of Theorem 2.16, we can prove the Proposition 2.17 below.

Proposition 2.17. Let the assumptions $(\mathrm{H} 0)$-to-(H4) be in force, and let $\mathcal{G}_{\mathcal{L}}$ be the Green's operator for $\mathcal{L}$ (thought of as an operator from $C(\overline{\mathcal{O}}, \mathbb{R})$ into itself).

Then, the following facts hold true:

(i) the spectral radius $r\left(\mathcal{G}_{\mathcal{L}}\right)$ of $\mathcal{G}_{\mathcal{L}}$ is strictly positive;

(ii) there exists a non-negative $u_{0} \in C^{1, \alpha}(\overline{\mathcal{O}}, \mathbb{R}) \backslash\{0\}$ such that

$$
\mathcal{G}_{\mathcal{L}}\left(u_{0}\right)=r\left(\mathcal{G}_{\mathcal{L}}\right) u_{0}
$$

Proof. (i) On account of Theorem 2.16, it is possible to find a real number $\sigma>0$ and a function $u_{\sigma} \in W^{1,2}(\mathcal{O}) \backslash\{0\}$ such that

$$
\left\{\begin{array}{l}
\mathcal{L} u=\sigma u \quad \text { in } \mathcal{O}, \\
\left.u\right|_{\partial \mathcal{O}}=0,
\end{array}\right.
$$

On the other hand, by applying the classical Elliptic Regularity Theory to $\mathcal{L}_{\sigma}:=\mathcal{L}-\sigma$ (see, e.g., [12, Corollary 8.35]), one can find a function $\hat{u}_{\sigma} \in C^{1, \alpha}(\overline{\mathcal{O}}, \mathbb{R})$ such that

$$
\hat{u}_{\sigma} \equiv u_{\sigma} \text { a.e. on } \mathcal{O}
$$

as a consequence, by the very definition of $\mathcal{G}_{\mathcal{L}}$ we infer that

$$
\mathcal{G}_{\mathcal{L}}\left(\hat{u}_{\sigma}\right)=\frac{1}{\sigma} \hat{u}_{\sigma}
$$

This proves that $\lambda:=1 / \sigma>0$ lays in the (point-wise) spectrum of $\mathcal{G}_{\mathcal{L}}$ (thought of as an operator from $C(\overline{\mathcal{O}}, \mathbb{R})$ into itself), and thus $r\left(\mathcal{G}_{\mathcal{L}}\right)>0$.

(ii) First of all, since $C^{1}(\overline{\mathcal{O}}, \mathbb{R})$ is continuously embedded in $C(\overline{\mathcal{O}}, \mathbb{R})$, we straightforwardly derive from Proposition 2.7 (ii) that $\mathcal{G}_{\mathcal{L}}$ is compact from $C(\overline{\mathcal{O}}, \mathbb{R})$ into itself; moreover, if we 
denote by $V_{0}$ the convex cone in $C(\overline{\mathcal{O}}, \mathbb{R})$ defined as

$$
V_{0}:=C\left(\overline{\mathcal{O}}, \mathbb{R}^{+}\right)=\{u \in C(\overline{\mathcal{O}}, \mathbb{R}): u \geq 0 \text { on } \mathcal{O}\}
$$

we know from Proposition 2.7 (iii) that $\mathcal{G}_{\mathcal{L}}\left(V_{0}\right) \subseteq V_{0}$. Since, obviously, $V_{0}-V_{0}$ is dense in $C(\overline{\mathcal{O}}, \mathbb{R})$ and since, by statement $(\mathrm{i})$, the spectral radius $r\left(\mathcal{G}_{\mathcal{L}}\right)$ of $\mathcal{G}_{\mathcal{L}}$ is strictly positive, we are entitled to apply Krein-Rutman's Theorem, ensuring that $r\left(\mathcal{G}_{\mathcal{L}}\right)$ is an eigenvalue of $\mathcal{G}_{\mathcal{L}}$ with positive eigenvector: this means that there exists $u_{0} \in V_{0} \backslash\{0\}$ such that

$$
\mathcal{G}_{\mathcal{L}}\left(u_{0}\right)=r\left(\mathcal{G}_{\mathcal{L}}\right) u_{0} \Longleftrightarrow u_{0}=\frac{1}{r\left(\mathcal{G}_{\mathcal{L}}\right)} \mathcal{G}_{\mathcal{L}}\left(u_{0}\right) \text {. }
$$

Now, since $u_{0} \in V_{0} \backslash\{0\}$, we have $u_{0} \geq 0$ and $u \not \equiv 0$ on $\mathcal{O}$; moreover, reminding that $\mathcal{G}_{\mathcal{L}}$ maps $C(\overline{\mathcal{O}}, \mathbb{R})$ into $C^{1, \alpha}(\overline{\mathcal{O}}, \mathbb{R})$ (see $(\underline{2.9})$ ), we derive that $u_{0} \in C^{1, \alpha}(\overline{\mathcal{O}}, \mathbb{R})$. Gathering together all these facts, we conclude that $u_{0} \in C^{1, \alpha}(\overline{\mathcal{O}}, \mathbb{R}) \backslash\{0\}$ and that $u \geq 0$ on $\overline{\mathcal{O}}$, as desired.

\section{EXISTENCE AND NON-EXISTENCE RESULTS}

In this Section we study the solvability of the following system of second order elliptic differential equations subject to functional BCs

$$
\left\{\begin{array}{lll}
\mathcal{L}_{k} u_{k}=\lambda_{k} f_{k}\left(x, u_{1}, \ldots, u_{m}, \nabla u_{1}, \ldots, \nabla u_{m}\right) & \text { in } \mathcal{O} & (k=1,2, \ldots, m), \\
u_{k}(x)=\eta_{k} \zeta_{k}(x) h_{k}\left[u_{1}, \ldots, u_{m}\right] & \text { for } x \in \partial \mathcal{O} & (k=1,2, \ldots, m),
\end{array}\right.
$$

where, as in the Introduction, $m \geq 1$ is a fixed natural number, $\mathcal{O} \subseteq \mathbb{R}^{n}$ is an open set and $\mathcal{L}_{1}, \ldots, \mathcal{L}_{m}$ are uniformly elliptic PDOs on $\mathcal{O}$ as in Section 2. To be more precise, we suppose that

(I) $\mathcal{O}$ is bounded, connected and of class $C^{1, \alpha}$ for some $\alpha \in(0,1)$;

(II) for every fixed $k=1, \ldots, m$, the differential operator $\mathcal{L}_{k}$ satisfies assumptions (H1)to-(H3) introduced in Section 2, that is,

(*) $\mathcal{L}_{k}$ takes the divergence form (2.1), i.e.,

$$
\mathcal{L}_{k} u:=-\sum_{i, j=1}^{n} \partial_{x_{i}}\left(a_{i, j}^{(k)}(x) \partial_{x_{j}} u+b_{i}^{(k)}(x) u\right)+\sum_{i=1}^{n} c_{i}^{(k)}(x) \partial_{x_{i}} u+d^{(k)}(x) u
$$

(*) the coefficient functions of $\mathcal{L}_{k}$ belong to $C^{1, \alpha}(\overline{\mathcal{O}}, \mathbb{R})$;

$(*)$ the matrix $A^{(k)}(x):=\left(a_{i, j}^{(k)}(x)\right)_{i, j}$ is symmetric for any $x \in \mathcal{O}$;

$(*) \mathcal{L}_{k}$ is uniformly elliptic in $\mathcal{O}$, i.e., there exists $\Lambda_{k}>0$ such that

$$
\frac{1}{\Lambda_{k}}\|\xi\|^{2} \leq \sum_{i, j=1}^{n} a_{i, j}^{(k)}(x) \xi_{i} \xi_{j} \leq \Lambda_{k}\|\xi\|^{2} \quad \text { for any } x \in \mathcal{O} \text { and } \xi \in \mathbb{R}^{n} \backslash\{0\}
$$

(*) for every non-negative function $\varphi \in C_{0}^{\infty}(\mathcal{O}, \mathbb{R})$ one has

$$
\int_{\mathcal{O}}\left(d^{(k)} \varphi+\sum_{i=1}^{n} b_{i}^{(k)} \partial_{x_{i}} \varphi\right) \mathrm{d} x, \quad \int_{\mathcal{O}}\left(d^{(k)} \varphi+\sum_{i=1}^{n} c_{i}^{(k)} \partial_{x_{i}} \varphi\right) \mathrm{d} x \geq 0 .
$$


Furthermore, for every fixed $k=1, \ldots, m$ we also assume that

(III) $f_{k}$ is a real-valued function defined on $\overline{\mathcal{O}} \times \mathbb{R}^{m} \times \mathbb{R}^{n m}$;

(IV) $h_{k}$ is a real-valued operator defined on the space $C^{1}\left(\overline{\mathcal{O}}, \mathbb{R}^{m}\right)$;

(V) $\zeta_{k} \in C^{1, \alpha}(\overline{\mathcal{O}}, \mathbb{R})$ and $\zeta_{k} \geq 0$ on $\mathcal{O}$;

(VI) $\lambda_{k}, \eta_{k}$ are non-negative real parameters.

Throughout the sequel, if $u_{1}, \ldots, u_{m}$ are real-valued functions defined on $\mathcal{O}$, we set

$$
\mathbf{u}(x):=\left(u_{1}(x), \ldots, u_{m}(x)\right) \quad(x \in \mathcal{O}) .
$$

If, in addition, $\mathbf{u} \in C^{1}\left(\mathcal{O}, \mathbb{R}^{m}\right)$ (that is, $u_{1}, \ldots, u_{m} \in C^{1}(\mathcal{O}, \mathbb{R})$ ), we define

$$
D \mathbf{u}(x):=\left(\nabla u_{1}(x), \ldots, \nabla u_{m}(x)\right) \quad(x \in \mathcal{O}) .
$$

Now, in view of assumptions (I)-(II), all the results presented in Section 2 can be applied to each operator $\mathcal{L}_{k}$ (for a fixed $k=1, \ldots, m$ ); in particular, for every $\mathfrak{f} \in C(\overline{\mathcal{O}}, \mathbb{R}$ ) there exists a unique solution $u_{\mathfrak{f}} \in C^{1, \alpha}(\overline{\mathcal{O}}, \mathbb{R})$ of the Poisson problem

$$
\left\{\begin{array}{l}
\mathcal{L}_{k} u=\mathfrak{f} \quad \text { in } \mathcal{O}, \\
\left.u\right|_{\partial \mathcal{O}}=0 .
\end{array}\right.
$$

Furthermore, since the function $\zeta_{k}$ belongs to $C^{1, \alpha}(\overline{\mathcal{O}}, \mathbb{R})$ (see assumption $(\mathrm{V})$ ), there exists a unique solution $\gamma_{k} \in C^{1, \alpha}(\overline{\mathcal{O}}, \mathbb{R})$ of the Dirichlet problem

$$
\left\{\begin{array}{l}
\mathcal{L}_{k} u=0 \quad \text { in } \mathcal{O}, \\
\left.u\right|_{\partial \mathcal{O}}=\zeta_{k} .
\end{array}\right.
$$

We then denote by $\mathcal{G}_{k}$ the Green's operator $\mathcal{G}_{\mathcal{L}_{k}}$ for $\mathcal{L}_{k}$ defined in (2.9), and we indicate by $g_{k}$ the Green's function $g_{\mathcal{L}_{k}}$ for the operator $\mathcal{G}_{k}$ defined through Theorem 2.8. We remind that, if $\mathfrak{f} \in C(\overline{\mathcal{O}}, \mathbb{R})$ is arbitrary fixed, $\mathcal{G}_{k}(\mathfrak{f})$ is the unique solution in $C^{1, \alpha}(\overline{\mathcal{O}}, \mathbb{R})$ of the Poisson problem (3.2); moreover, we have the representation formulas

$$
\mathcal{G}_{k}(\mathfrak{f})(x)=\int_{\mathcal{O}} g_{k}(y ; x) \mathfrak{f}(y) \mathrm{d} y \quad \text { and } \quad \partial_{x_{i}} \mathcal{G}_{k}(\mathfrak{f})(x)=\int_{\mathcal{O}} \partial_{x_{i}} g_{k}(y ; x) \mathfrak{f}(y) \mathrm{d} y,
$$

holding true for a.e. $x \in \mathcal{O}$ and any $i=1, \ldots, n$ (see Theorem 2.8 and Proposition 2.14).

Finally, according to Proposition 2.17, we denote by $r_{k}=r\left(\mathcal{G}_{k}\right)>0$ the spectral radius of the operator $\mathcal{G}_{k}$ (thought of as an operator from $C^{1}(\overline{\mathcal{O}}, \mathbb{R})$ into itself) and we fix once and for all a function $\varphi_{k} \in C^{1, \alpha}(\overline{\mathcal{O}}, \mathbb{R}) \backslash\{0\}$ such that (setting $\mu_{k}:=1 / r_{k}$ )

$$
\varphi_{k}=\mu_{k} \mathcal{G}_{k}\left(\varphi_{k}\right) \quad \text { and } \quad \varphi_{k} \geq 0 \text { on } \mathcal{O} \text {. }
$$

Now that we have properly introduced all the "mathematical objects" appearing in the problem (3.1), it is opportune to define what we mean by a solution of this problem. 
To this end, we first fix some notation. For every index $k \in\{1, \ldots, m\}$, we denote by $\mathcal{F}_{k}$ the so-called superposition (Nemytskii) operator associated with $f_{k}$, that is,

$$
\mathcal{F}_{k}: C^{1}\left(\overline{\mathcal{O}}, \mathbb{R}^{m}\right) \rightarrow C(\overline{\mathcal{O}}, \mathbb{R}), \quad \mathcal{F}_{k}(\mathbf{u}):=f_{k}(x, \mathbf{u}, D \mathbf{u}) .
$$

Moreover, we consider the operators $\mathcal{T}, \Gamma: C^{1}\left(\overline{\mathcal{O}}, \mathbb{R}^{m}\right) \rightarrow C^{1}\left(\overline{\mathcal{O}}, \mathbb{R}^{m}\right)$ defined by

$$
\mathcal{T}(\mathbf{u})=\left(\lambda_{k}\left(\mathcal{G}_{k} \circ \mathcal{F}_{k}\right)(\mathbf{u})\right)_{k=1, \ldots, m} \quad \text { and } \quad \Gamma(\mathbf{u}):=\left(\eta_{k} \gamma_{k}(x) h_{k}[\mathbf{u}]\right)_{k=1, \ldots, m} .
$$

We can now give the definition of solution of the problem (3.1).

Definition 3.1. We say that a function $\mathbf{u} \in C^{1}\left(\overline{\mathcal{O}}, \mathbb{R}^{m}\right)$ is a weak solution of the system (3.1) if $\mathbf{u}$ is a fixed point of the operator $\mathcal{T}+\Gamma$, that is,

$$
\mathbf{u}=\mathcal{T}(\mathbf{u})+\Gamma(\mathbf{u})=\left(\lambda_{k}\left(\mathcal{G}_{k} \circ \mathcal{F}_{k}\right)(\mathbf{u})+\eta_{k} \gamma_{k}(x) h_{k}[\mathbf{u}]\right)_{k=1, \ldots, m} .
$$

If, in addition, the components of $\mathbf{u}$ are non-negative and $u_{j} \not \equiv 0$ for some $j$, we say that $\mathbf{u}$ is a nonzero positive solution of the system (3.1).

For our existence result, we make use of the following proposition that states the main properties of the classical fixed point index, for more details see [2, 16]. In what follows the closure and the boundary of subsets of a cone $\hat{P}$ are understood to be relative to $\hat{P}$.

Proposition 3.2. Let $X$ be a real Banach space and let $\hat{P} \subset X$ be a cone. Let $D$ be an open bounded set of $X$ with $0 \in D \cap \hat{P}$ and $\overline{D \cap \hat{P}} \neq \hat{P}$. Assume that $T: \overline{D \cap \hat{P}} \rightarrow \hat{P}$ is a compact operator such that $x \neq T(x)$ for $x \in \partial(D \cap \hat{P})$.

Then the fixed point index $i_{\hat{P}}(T, D \cap \hat{P})$ has the following properties:

(i) If there exists $e \in \hat{P} \backslash\{0\}$ such that $x \neq T(x)+\sigma e$ for all $x \in \partial(D \cap \hat{P})$ and all $\sigma>0$, then $i_{\hat{P}}(T, D \cap \hat{P})=0$.

(ii) If $T(x) \neq \sigma x$ for all $x \in \partial(D \cap \hat{P})$ and all $\sigma>1$, then $i_{\hat{P}}(T, D \cap \hat{P})=1$.

(iii) Let $D^{1}$ be open bounded in $X$ such that $\left(\overline{D^{1} \cap \hat{P}}\right) \subset(D \cap \hat{P})$. If $i_{\hat{P}}(T, D \cap \hat{P})=1$ and $i_{\hat{P}}\left(T, D^{1} \cap \hat{P}\right)=0$, then $T$ has a fixed point in $(D \cap \hat{P}) \backslash\left(\overline{D^{1} \cap \hat{P}}\right)$. The same holds if $i_{\hat{P}}(T, D \cap \hat{P})=0$ and $i_{\hat{P}}\left(T, D^{1} \cap \hat{P}\right)=1$.

We can now state a result regarding the existence of positive solutions for the system (3.1).

In the sequel, we will consider on the space $\mathbb{R}^{s}$ (where $s$ will be either $m, n$ or $m n$ ) the following maximum norm

$$
|\mathbf{v}|:=\max _{i=1, \ldots, s}\left|v_{i}\right| \quad\left(\text { if } \mathbf{v}=\left(v_{1}, \ldots, v_{s}\right)\right) .
$$

We will work in the Banach space $C\left(\overline{\mathcal{O}}, \mathbb{R}^{m}\right)$ endowed with the norm

$$
\|\mathbf{z}\|_{\infty}=\max _{x \in \overline{\mathcal{O}}}|\mathbf{z}(x)|:=\max \left\{\left\|z_{1}\right\|_{\infty}, \ldots,\left\|z_{m}\right\|_{\infty}\right\}
$$


where $\mathbf{z}=\left(z_{1}, \ldots, z_{m}\right) \in C\left(\overline{\mathcal{O}}, \mathbb{R}^{m}\right)$, compare also with (2.4). Moreover, we will consider the Banach space $C^{1}\left(\overline{\mathcal{O}}, \mathbb{R}^{m}\right)$ endowed with the norm

$$
\begin{aligned}
\|\mathbf{u}\|_{C^{1}\left(\overline{\left.\mathcal{O}, \mathbb{R}^{m}\right)}\right.} & :=\max \left\{\max _{k=1,2, \ldots, m}\left\|u_{k}\right\|_{\infty}, \max _{k=1,2, \ldots, m}\left\|\nabla u_{k}\right\|_{\infty}\right\} \\
& =\max \left\{\left\|u_{k}\right\|_{\infty},\left\|\partial_{x_{l}} u_{k}\right\|_{\infty}: k=1, \ldots, m \text { and } l=1, \ldots, n\right\}
\end{aligned}
$$

notice that (3.6) reduces to (2.5) when $m=1$. Given a finite sequence $\varrho=\left\{\rho_{k}\right\}_{k=1}^{m} \subseteq$ $(0,+\infty)$, we define

$$
I(\varrho)=\prod_{k=1}^{m}\left[0, \rho_{k}\right] \quad \text { and } \quad R(\varrho)=\prod_{k=1}^{m} R_{\rho_{k}}
$$

where $R_{\rho}=\left\{\mathbf{v} \in \mathbb{R}^{n}:|\mathbf{v}| \leq \rho\right\}$ (for $t>0$ ); we also introduce, with abuse of notation, the sets

$$
\begin{aligned}
& P:=\left\{\mathbf{u} \in C^{1}\left(\overline{\mathcal{O}}, \mathbb{R}^{m}\right): u_{k} \geq 0 \text { on } \mathcal{O} \text { for every } k=1, \ldots, m\right\} \quad \text { and } \\
& P(\varrho)=\left\{\mathbf{u} \in C^{1}\left(\overline{\mathcal{O}}, \mathbb{R}^{m}\right): \mathbf{u}(x) \in I(\varrho) \text { and } D \mathbf{u}(x) \in R(\varrho) \text { for all } x \in \overline{\mathcal{O}}\right\} \subseteq P .
\end{aligned}
$$

Theorem 3.3. Let the assumptions (I)-to-(VI) be in force. Moreover, let us suppose that one can find a finite sequence $\varrho=\left\{\rho_{k}\right\}_{k=1}^{m} \subseteq(0, \infty)$ satisfying the following hypotheses:

(a) For every $k=1, \ldots, m$, one has that

$(\mathrm{a})_{1} f_{k}$ continuous and non-negative on $\overline{\mathcal{O}} \times I(\varrho) \times R(\varrho)$;

$(\mathrm{a})_{2} h_{k}$ continuous, non-negative and bounded on $P(\varrho)$.

(b) There exist $\delta \in(0,+\infty), k_{0} \in\{1,2, \ldots, m\}$ and $\rho_{0} \in\left(0, \min _{k=1, \ldots, m} \rho_{k}\right)$ such that

$$
f_{k_{0}}(x, \mathbf{z}, \mathbf{w}) \geq \delta z_{k_{0}} \quad \text { for every }(x, \mathbf{z}, \mathbf{w}) \in \overline{\mathcal{O}} \times I_{0} \times B_{0},
$$

where $I_{0}:=\prod_{i=1}^{m}\left[0, \rho_{0}\right]$ and $R_{0}:=\prod_{k=1}^{m} R_{\rho_{0}}$.

(c) Setting, for every $k=1, \ldots, m$,

$$
\begin{aligned}
& M_{k}:=\max \left\{f_{k}(x, \mathbf{z}, \mathbf{w}):(x, \mathbf{z}, \mathbf{w}) \in \overline{\mathcal{O}} \times I(\varrho) \times R(\varrho)\right\} \quad \text { and } \\
& H_{k}:=\sup _{\mathbf{u} \in P(\varrho)} h_{k}[\mathbf{u}] .
\end{aligned}
$$

the following inequalities are satisfied:

(c) ${ }_{1} \mu_{k_{0}} \leq \delta \lambda_{k_{0}}$;

(c) ${ }_{2} \lambda_{k} M_{k}\left\|\mathcal{G}_{k}(\hat{1})\right\|_{\infty}+\eta_{k} H_{k}\left\|\gamma_{k}\right\|_{\infty} \leq \rho_{k}$;

$(\mathrm{c})_{3}$ for any $l=1, \ldots, n$ we have $\lambda_{k} M_{k} G_{k, l}+\eta_{k} H_{k}\left\|\partial_{x_{l}} \gamma_{k}\right\|_{\infty} \leq \rho_{k}$, where

$$
G_{k, l}:=\sup _{x \in \mathcal{O}} \int_{\mathcal{O}}\left|\partial_{x_{l}} g_{k}(y ; x)\right| \mathrm{d} y \quad \text { (see Lemma 2.12). }
$$


Then the system (3.1) has a non-zero positive weak solution $\mathbf{u} \in C^{1}\left(\overline{\mathcal{O}}, \mathbb{R}^{m}\right)$ such that

$$
\|\mathbf{u}\|_{C^{1}\left(\overline{\mathcal{O}}, \mathbb{R}^{m}\right)} \geq \rho_{0} \quad \text { and } \quad\left\|u_{k}\right\|_{\infty} \leq \rho_{k} \text { for every } k=1, \ldots, m .
$$

Proof. For the sake of readability, we split the proof into different steps.

SteP I: We first prove that the operator $\mathcal{A}:=\mathcal{T}+\Gamma$ maps $P(\varrho)$ into $P$.

To this end, let $\mathbf{u} \in P(\varrho)$ and let $k \in\{1, \ldots, m\}$ be fixed. Since $\mathbf{u} \in P(\varrho)$, from assumption (a) $)_{2}$ we derive that $h_{k}[\mathbf{u}] \geq 0$; moreover, since $\gamma_{k} \geq 0$ on $\overline{\mathcal{O}}$ (see Proposition 2.7-(iii)) and since, by assumption (VI), $\eta_{k} \geq 0$, we get

$$
\Gamma_{k}(\mathbf{u})(x)=\eta_{k} \gamma_{k}(x) h_{k}[\mathbf{u}] \geq 0 \text { for all } x \in \overline{\mathcal{O}} .
$$

On the other hand, since $\mathbf{u} \in P(\varrho)$, by assumption (a) $)_{1}$ we also have that

$$
\mathcal{F}_{k}(\mathbf{u})(x)=f_{k}(x, \mathbf{u}(x), D \mathbf{u}(x)) \geq 0 \quad \text { for all } x \in \overline{\mathcal{O}}
$$

as a consequence, from Proposition 2.7.(iii) we derive that $\mathcal{G}_{k}\left(\mathcal{F}_{k}(\mathbf{u})\right) \geq 0$ on $\overline{\mathcal{O}}$. Finally, since $\lambda_{k} \geq 0$ (by assumption (IV)), we get

$$
\mathcal{T}_{k}(\mathbf{u})(x)=\lambda_{k} \mathcal{G}_{k}\left(\mathcal{F}_{k}(\mathbf{u})(x)\right) \geq 0 \quad \text { for every } x \in \overline{\mathcal{O}} .
$$

By (3.13), (3.14) and the arbitrariness of $k$, we conclude that $\mathcal{A}(P(\varrho)) \subseteq P$.

Step II: We now prove that $\mathcal{A}: P(\varrho) \rightarrow P$ is compact. To this end, let $\left\{\mathbf{u}_{j}\right\}_{j \in \mathbb{N}}$ be a bounded sequence in $P(\varrho)$, and let $k \in\{1, \ldots, m\}$ be fixed. Since $h_{k}$ is non-negative and bounded on $P(\varrho)$ (see assumption $\left.(\mathrm{a})_{2}\right)$, the sequence $\left\{h_{k}\left[\mathbf{u}_{j}\right]\right\}_{j}$ is bounded in $(0, \infty)$; as a consequence, there exists $\theta_{0} \in[0, \infty)$ such that (up to a sub-sequence)

$$
\lim _{j \rightarrow \infty} \Gamma_{k}\left(\mathbf{u}_{j}\right)=\eta_{k} \gamma_{k}(x) \theta_{0} \quad \text { in } C^{1}(\overline{\mathcal{O}}, \mathbb{R}) .
$$

On the other hand, since $\left\{\mathbf{u}_{j}\right\}_{j} \subseteq P(\varrho)$ and since $f_{k}$ is continuous on $\overline{\mathcal{O}} \times I(\varrho) \times R(\varrho)$ (see assumption $\left.(\mathrm{a})_{1}\right)$, we have (using the notation in (3.10) $)$

$$
\left\|\mathcal{F}\left(\mathbf{u}_{j}\right)\right\|_{\infty} \leq M_{k} \quad \text { for every } j \in \mathbb{N} \text {. }
$$

As a consequence, since the operator $\mathcal{G}_{k}$ is compact (as an operator from $C(\overline{\mathcal{O}}, \mathbb{R}$ ) into $C^{1}(\overline{\mathcal{O}}, \mathbb{R})$, see Proposition 2.7-(ii)), it is possible to find a function $w_{k} \in C^{1}(\overline{\mathcal{O}}, \mathbb{R})$ such that (again by possibly passing to a sub-sequence)

$$
\lim _{j \rightarrow \infty} \mathcal{T}_{k}\left(\mathbf{u}_{j}\right)=\lim _{j \rightarrow \infty}\left(\lambda_{k} \mathcal{G}_{k}\left(\mathcal{F}_{k}\left(\mathbf{u}_{j}\right)\right)\right)=\lambda_{k} w_{k} \quad \text { in } C^{1}(\overline{\mathcal{O}}, \mathbb{R}) .
$$

Gathering together (3.15), (3.16) and (3.6), we infer that (up to a suitable sub-sequence)

$$
\lim _{j \rightarrow \infty} \mathcal{A}\left(\mathbf{u}_{j}\right)=\left(\lambda_{k} w_{k}+\eta_{k} \gamma_{k} \theta_{0}\right)_{k=1, \ldots, m}=: \widetilde{\mathbf{u}} \quad \text { in } C^{1}\left(\overline{\mathcal{O}}, \mathbb{R}^{m}\right) .
$$


Finally, since $\left\{\mathcal{A}\left(\mathbf{u}_{j}\right)\right\}_{j} \subseteq P$ (by Step I) and since $P$ is closed, we conclude that $\widetilde{\mathbf{u}} \in P$; this proves the compactness of $\mathcal{A}$ (as an operator from $P(\varrho)$ to $P$ ).

To proceed further, we consider the set $P_{0} \subseteq C^{1}\left(\overline{\mathcal{O}}, \mathbb{R}^{m}\right)$ defined as follows:

$$
P_{0}=\left\{\mathbf{u} \in C^{1}\left(\overline{\mathcal{O}}, \mathbb{R}^{m}\right): \mathbf{u}(x) \in I_{0} \text { and } D \mathbf{u}(x) \in R_{0} \text { for all } x \in \overline{\mathcal{O}}\right\} \subseteq P(\varrho),
$$

where $I_{0}$ and $B_{0}$ are as in assumption (b). Now, if the operator $\mathcal{A}=\mathcal{T}+\Gamma$ has a fixed point $\mathbf{u}_{0} \in \partial P_{0} \cup \partial P(\varrho)$ (where the boundaries are both relative to $P$ ), then $\mathbf{u}_{0}$ is a solution of problem (3.1) satisfying (3.12), and the theorem is proved.

If, instead, $\mathcal{A}$ is fixed-point free on $\partial P_{0} \cup \partial P(\varrho)$, the fixed-point indexes

$$
i_{P}\left(\mathcal{A}, \operatorname{int}\left(P_{0}\right) \cap P\right) \quad \text { and } \quad i_{P}(\mathcal{A}, \operatorname{int}(P(\varrho)) \cap P)
$$

are well-defined. Assuming this last possibility, we consider the following steps.

STEP III: In this step we prove the following fact:

$$
i_{P}(\mathcal{A}, \operatorname{int}(P(\varrho)) \cap P)=1 .
$$

According to Proposition 3.2.(ii), to prove (3.17) it suffices to show that

$$
\mathcal{A}(\mathbf{u}) \neq \sigma \mathbf{u} \quad \text { for every } \mathbf{u} \in \partial P(\varrho) \text { and every } \sigma>1,
$$

To establish (3.18) we argue by contradiction, and we suppose that there exist a function $\mathbf{u} \in \partial P(\varrho)$ and a real $\sigma>1$ such that

$$
\sigma \mathbf{u}=\mathcal{A}(\mathbf{u})=\mathcal{T}(\mathbf{u})+\Gamma(\mathbf{u}) .
$$

Since $\mathbf{u} \in \partial P(\varrho)$, there exists an index $k \in\{1, \ldots, m\}$ such that either

$$
\left\|u_{k}\right\|_{\infty}=\rho_{k} \text { or }\left\|\nabla u_{k}\right\|_{\infty}=\rho_{k} .
$$

We then distinguish these two cases.

- $\left\|u_{k}\right\|_{\infty}=\rho_{k}$. In this case, by exploiting assumption (a) ${ }_{1}$ and (3.10), we have

$$
0 \leq \mathcal{F}_{k}(\mathbf{u})(x)=f_{k}(x, \mathbf{u}(x), D \mathbf{u}(x)) \leq M_{k} \quad \text { for all } x \in \overline{\mathcal{O}} ;
$$

from this, we derive the following chain of inequalities:

$$
\begin{aligned}
& \sigma u_{k}(x)=\lambda_{k} \mathcal{G}_{k}\left(\mathcal{F}_{k}(\mathbf{u})\right)(x)+\eta_{k} \gamma_{k}(x) h_{k}[\mathbf{u}] \\
& \text { (since } \mathcal{G}_{k}\left(M_{k} \hat{1}-\mathcal{F}_{k}(\mathbf{u})\right) \geq 0 \text {, see (3.19) and Proposition 2.7-(iii)) } \\
& \leq \lambda_{k} \mathcal{G}_{k}\left(M_{k} \hat{1}\right)(x)+\eta_{k} \gamma_{k}(x) h_{k}[\mathbf{u}] \\
& \text { (since } \mathbf{u} \in \partial P(\varrho) \subseteq P(\varrho) \text {, see (3.10)) } \\
& \leq\left\|\lambda_{k} \mathcal{G}_{k}\left(M_{k} \hat{1}\right)\right\|_{\infty}+\left\|\eta_{k} H_{k} \gamma_{k}\right\|_{\infty} \\
& =\lambda_{k} M_{k}\left\|\mathcal{G}_{k}(\hat{1})\right\|_{\infty}+\eta_{k} H_{k}\left\|\gamma_{k}\right\|_{\infty} \leq \rho_{k} \quad \text { (see assumption }(\mathrm{c})_{2} \text { ). }
\end{aligned}
$$


As a consequence, by taking the supremum for $x \in \overline{\mathcal{O}}$ in 3.20 (and by reminding that $\mathbf{u} \in \partial P(\varrho) \subseteq P(\varrho))$, we then obtain

$$
\sup _{x \in \overline{\mathcal{O}}}\left|\sigma u_{k}(x)\right| \leq \sigma \rho_{k} \leq \rho_{k},
$$

which is clearly a contradiction (since $\sigma>1$ ).

- $\left\|\nabla u_{k}\right\|_{\infty}=\rho_{k}$. In this case, by the very definition of $\|\cdot\|_{\infty}$, there exists $l \in\{1, \ldots, n\}$ such that $\left\|\partial_{x_{l}} u_{k}\right\|_{\infty}=\rho_{k}$. Moreover, by Proposition 2.14 we have

$$
\sigma \partial_{x_{l}} u_{k}(x)=\lambda_{k} \int_{\mathcal{O}} \partial_{x_{l}} g_{k}(y ; x) f_{k}(x, \mathbf{u}(y), D \mathbf{u}(y)) \mathrm{d} y+\eta_{k} \partial_{x_{l}} \gamma_{k}(x) h_{k}[\mathbf{u}]
$$

for a.e. $x \in \mathcal{O}$. By means of this representation formula, we then obtain

$$
\begin{aligned}
\sigma \mid \partial_{x_{l}} & u_{k}(x) \mid \\
& \leq \lambda_{k} \int_{\mathcal{O}}\left|\partial_{x_{l}} g_{k}(y ; x) f_{k}(x, \mathbf{u}(y), D \mathbf{u}(y))\right| \mathrm{d} y+\eta_{k} h_{k}[\mathbf{u}]\left|\partial_{x_{l}} \gamma_{k}(x)\right| \\
& (\text { since } \mathbf{u} \in \partial P(\varrho) \subseteq P(\varrho), \text { see also }(\underline{3.19})) \\
& \leq \lambda_{k} M_{k} \int_{\mathcal{O}}\left|\partial_{x_{l}} g_{k}(y ; x)\right| \mathrm{d} y+\eta_{k} H_{k}\left|\partial_{x_{l}} \gamma_{k}(x)\right| \\
& \leq \lambda_{k} M_{k} G_{k, l}+\eta_{k} H_{k}\left\|\partial_{x_{l}} \gamma_{k}\right\|_{\infty} \quad(\text { see (3.11) })
\end{aligned}
$$

As a consequence, by taking the supremum for $x \in \overline{\mathcal{O}}$ in (3.20) (and by reminding that $\left.\left\|\partial_{x_{l}} u_{k}\right\|_{\infty}=\rho_{k}\right)$, from assumption $(\mathrm{c})_{3}$ we infer that

$$
\sup _{x \in \overline{\mathcal{O}}}\left(\sigma\left|\partial_{x_{l}} u_{k}(x)\right|\right)=\sigma \rho_{k} \leq \lambda_{k} M_{k} G_{k, l}+\eta_{k} H_{k}\left\|\partial_{x_{l}} \gamma_{k}\right\|_{\infty} \leq \rho_{k}
$$

which is clearly a contradiction (as $\sigma>1$ ).

This completes the demonstration of (3.18).

STEP IV: In this last step we prove the following fact:

$$
i_{P}\left(\mathcal{A}, \operatorname{int}\left(P_{0}\right) \cap P\right)=0 .
$$

According to Proposition 3.2-(i), to prove (3.21) it suffices to show that there exists a suitable function $e \in P \backslash\{0\}$ satisfying the property

$$
\mathcal{A}(\mathbf{u})+\sigma e \neq \mathbf{u} \quad \text { for every } \mathbf{u} \in \partial P_{0} \text { and every } \sigma>0 \text {. }
$$

To establish (3.22), we let $e:=\left(\varphi_{1}, \ldots, \varphi_{m}\right)$ (where $\varphi_{1}, \ldots, \varphi_{m}$ are as in (3.4)) and we argue by contradiction: we thus suppose that there exist $\mathbf{u} \in \partial P_{0}$ and $\sigma>0$ such that

$$
\mathbf{u}=\mathcal{A}(\mathbf{u})+\sigma e=\underset{20}{\mathcal{T}}(\mathbf{u})+\Gamma(\mathbf{u})+\sigma e .
$$


Since $\mathbf{u} \in \partial P_{0} \subseteq P_{0} \subseteq P(\varrho)$ (by definition of $P_{0}$, see assumption (b)), we know from Step I that $\mathcal{A}(\mathbf{u}) \in P$; as a consequence, if $k_{0}$ is as in assumption (b), we have

$$
u_{k_{0}}=\mathcal{A}(\mathbf{u})_{k_{0}}+\sigma \varphi_{k_{0}} \geq \sigma \varphi_{k_{0}} \quad \text { on } \overline{\mathcal{O}} \text {. }
$$

Furthermore, by exploiting once again assumption (b) we get

$$
\mathcal{F}_{k_{0}}(\mathbf{u})=f_{k_{0}}(x, \mathbf{u}(x), D \mathbf{u}(x)) \geq \delta u_{k_{0}}(x) \geq \delta \sigma \varphi_{k_{0}}(x) \quad \text { for all } x \in \overline{\mathcal{O}}
$$

Gathering together all these facts, for every $x \in \overline{\mathcal{O}}$ we have

$$
\begin{aligned}
u_{k_{0}}(x) & =\lambda_{k_{0}} \mathcal{G}_{k_{0}}\left(\mathcal{F}_{k_{0}}(\mathbf{u})\right)(x)+\eta_{k_{0}} \gamma_{k_{0}}(x) h_{k_{0}}[\mathbf{u}]+\sigma \varphi_{k_{0}}(x) \\
& \left(\text { since } \mathcal{G}_{k_{0}}\left(\mathcal{F}_{k_{0}}(\mathbf{u})-\delta \sigma \varphi_{k_{0}}\right) \geq 0, \text { see }(\underline{3.23})\right. \text { and Proposition 2.7-(iii)) } \\
& \geq \lambda_{k_{0}} \mathcal{G}_{k_{0}}\left(\delta \sigma \varphi_{k_{0}}\right)(x)+\sigma \varphi_{k_{0}}(x) \\
& \left(\text { since } \varphi_{k_{0}} \text { is an eigenfunction of } \mathcal{G}_{k_{0}}, \text { see (3.4) }\right) \\
& \left.=\frac{\delta \lambda_{k_{0}}}{\mu_{k_{0}}} \cdot \sigma \varphi_{k_{0}}(x)+\sigma \varphi_{k_{0}}(x) \geq 2 \sigma \varphi_{k_{0}}(x) \quad \text { (see assumption }(\mathrm{c})_{1}\right) .
\end{aligned}
$$

By iterating the above argument, for every $x \in \overline{\mathcal{O}}$ we get

$$
u_{k_{0}}(x) \geq p \sigma \varphi_{k_{0}}(x) \quad \text { for every } p \in \mathbb{N},
$$

but this is contradiction with the boundedness of $u_{k_{0}} \in C^{1}(\overline{\mathcal{O}}, \mathbb{R})\left(\right.$ as $\left.\varphi_{k_{0}} \not \equiv 0\right)$.

We are now ready to conclude the proof of the theorem: in fact, by combining (3.17), (3.21) and Proposition 3.2-(iii), we infer the existence of a fixed point

$$
\mathbf{u}_{0} \in(\operatorname{int}(P(\varrho)) \cap P) \backslash P_{0}
$$

of $\mathcal{A}=\mathcal{T}+\Gamma$; thus, $\mathbf{u}_{0}$ is a solution of (3.1) satisfying (3.12).

Remark 3.4. Let the assumption and the notation of Theorem 3.3 do apply. We have already pointed out that, since $\zeta_{1}, \ldots, \zeta_{m} \in C^{1, \alpha}(\overline{\mathcal{O}}, \mathbb{R})$ (see assumption $(\mathrm{V})$ ), one has

$$
\gamma_{k} \in C^{1, \alpha}(\overline{\mathcal{O}}, \mathbb{R}) \quad \text { for every } k=1, \ldots, m \text {. }
$$

As a consequence, the operator $\Gamma$ maps $C^{1}\left(\overline{\mathcal{O}}, \mathbb{R}^{m}\right)$ into $C^{1, \alpha}\left(\overline{\mathcal{O}}, \mathbb{R}^{m}\right)$. On the other hand, since the operators $\mathcal{G}_{1}, \ldots, \mathcal{G}_{m}$ map $C(\overline{\mathcal{O}}, \mathbb{R})$ into $C^{1, \alpha}(\overline{\mathcal{O}}, \mathbb{R})$, we also have that

$$
\mathcal{T}\left(C^{1}\left(\overline{\mathcal{O}}, \mathbb{R}^{m}\right)\right) \subseteq C^{1, \alpha}\left(\overline{\mathcal{O}}, \mathbb{R}^{m}\right)
$$

Gathering together all these facts, we conclude that any weak solution of (3.1) (i.e., any fixed point of $\mathcal{A}=\mathcal{T}+\Gamma$ in $\left.C^{1}\left(\overline{\mathcal{O}}, \mathbb{R}^{m}\right)\right)$ is actually of class $C^{1, \alpha}$ on $\overline{\mathcal{O}}$.

An elementary argument yields the following non-existence result. 
Theorem 3.5. Let the assumptions (I)-to-(IV) be in force. Moreover, let us suppose that there exists a finite sequence $\varrho=\left\{\rho_{k}\right\}_{k=1}^{m} \subseteq(0, \infty)$ such that, for every $k=1, \ldots, m$, the following conditions hold:

(a) $f_{k}$ is continuous on $\overline{\mathcal{O}} \times I(\varrho) \times R(\varrho)$, and there exist $\tau_{k} \in(0,+\infty)$ such that

$$
0 \leq f_{k}(x, \mathbf{z}, \mathbf{w}) \leq \tau_{k} z_{k} \quad \text { for every }(x, \mathbf{z}, \mathbf{w}) \in \overline{\mathcal{O}} \times I(\varrho) \times R(\varrho)
$$

(b) $h_{k}$ is continuous on $P(\varrho)$ and there exist $\xi_{k} \in(0,+\infty)$ such that

$$
h_{k}[\mathbf{u}] \leq \xi_{k} \cdot\|\mathbf{u}\|_{\infty}, \quad \text { for every } u \in P(\varrho)
$$

(c) the following inequality holds:

$$
\lambda_{k} \tau_{k}\left\|\mathcal{G}_{k}(\hat{1})\right\|_{\infty}+\eta_{k} \xi_{k}\left\|\gamma_{k}\right\|_{\infty}<1
$$

Then the system (3.1) has at most the zero solution in $P(\varrho)$.

Proof. We argue by contradiction and we assume that (3.1) has a solution $\mathbf{u} \in P(\varrho) \backslash\{0\}$. According to Definition 3.1, this means that $\mathbf{u}$ is a fixed point of the operator $\mathcal{A}=\mathcal{T}+\Gamma$. Setting $\rho:=\|\mathbf{u}\|_{\infty}>0$, we let $j \in\{1,2, \ldots, m\}$ such that

$$
\left\|u_{j}\right\|_{\infty}=\rho
$$

For every $x \in \overline{\mathcal{O}}$, we then have

$$
0 \leq \mathcal{F}_{j}(\mathbf{u})(x)=f_{j}(x, \mathbf{u}(x), D \mathbf{u}(x)) \leq \tau_{j} u_{j}(x) \leq \tau_{j} \rho
$$

from this, we obtain

$$
\begin{aligned}
u_{j}(x) & =\lambda_{j} \mathcal{G}_{j}\left(\mathcal{F}_{j}(\mathbf{u})\right)(x)+\eta_{j} \gamma_{j}(x) h_{j}[\mathbf{u}] \\
& \left(\text { since } \mathcal{G}_{j}\left(\tau_{j} \rho \cdot \hat{1}-\mathcal{F}_{j}(\mathbf{u})\right) \geq 0, \text { see (13.26) and Proposition } 2.7 \text { (iii) }\right) \\
& \leq \lambda_{j} \mathcal{G}_{j}\left(\tau_{j} \rho \hat{1}\right)(x)+\eta_{j} \gamma_{j}(x) h_{j}[\mathbf{u}]
\end{aligned}
$$

(by assumption (b) and since $\|\mathbf{u}\|_{\infty}=\rho$ )

$$
\begin{aligned}
& \leq\left\|\lambda_{j} \mathcal{G}_{j}\left(\tau_{j} \rho \hat{1}\right)\right\|_{\infty}+\left\|\eta_{j} \xi_{j} \rho \gamma_{j}\right\|_{\infty} \\
& =\left(\lambda_{j} \tau_{j}\left\|\mathcal{G}_{k}(\hat{1})\right\|_{\infty}+\eta_{j} \xi_{j}\left\|\gamma_{j}\right\|_{\infty}\right) \rho .
\end{aligned}
$$

By taking the supremum in (3.27) for $x \in \overline{\mathcal{O}}$, from (3.24) and (3.25) we finally obtain

$$
\rho=\sup _{x \in \overline{\mathcal{O}}} u_{j}(x) \leq\left(\lambda_{j} \tau_{j}\left\|\mathcal{G}_{k}(\hat{1})\right\|_{\infty}+\eta_{j} \xi_{j}\left\|\gamma_{j}\right\|_{\infty}\right) \rho<\rho,
$$

a contradiction. Thus, problem (3.1) cannot have nonzero solutions in $P(\varrho)$. 


\section{EXAmples}

In this last section we present a couple of concrete examples illustrating the applicability of our main results, namely Theorems 3.3 and 3.5 .

Example 4.1. On Euclidean space $\mathbb{R}^{3}$, let us consider the following BVP

$$
\begin{cases}-\Delta u_{1}=\lambda_{1} e^{u_{1}}\left(1+\left|\nabla u_{2}\right|^{2}\right) & \text { in } B \\ -\Delta u_{2}=\lambda_{2}\left(16-u_{2}^{2}\right) \cos \left(\left\langle\nabla u_{1}, \nabla u_{2}\right\rangle\right) & \text { in } B \\ \left.u_{1}\right|_{\partial B}=\eta_{1}\left(u_{1}(0)+u_{2}(0)\right), & \\ \left.u_{2}\right|_{\partial B}=\eta_{2} \int_{\partial B_{1}} u_{1}\left(1-\left|\nabla u_{2}\right|^{2}\right) \mathrm{d} \sigma, & \end{cases}
$$

where $B$ is the Euclidean ball centered at 0 with radius 1 , and $|\cdot|$ is the max norm in $\mathbb{R}^{3}$, as in (3.5).

Obviously, this problem takes the form (3.1) with (here and throughout, we denote the points of $\mathbb{R}^{6}$ by $\mathbf{w}=\left(\mathbf{w}_{1}, \mathbf{w}_{2}\right)$, with $\left.\mathbf{w}_{1}, \mathbf{w}_{2} \in \mathbb{R}^{3}\right)$

(i) $\mathcal{O}:=B$;

(ii) $\mathcal{L}_{1}=\mathcal{L}_{2}=-\Delta$;

(iii) $f_{1}: \bar{B} \times \mathbb{R}^{2} \times \mathbb{R}^{6} \rightarrow \mathbb{R}, \quad f_{1}(x, \mathbf{z}, \mathbf{w})=e^{z_{1}}\left(1+\left|\mathbf{w}_{2}\right|^{2}\right)$;

(iv) $f_{2}: \bar{B} \times \mathbb{R}^{2} \times \mathbb{R}^{6} \rightarrow \mathbb{R}, \quad f_{2}(x, \mathbf{z}, \mathbf{w})=\left(16-z_{2}^{2}\right) \cos \left(\left\langle\mathbf{w}_{1}, \mathbf{w}_{2}\right\rangle\right)$;

(v) $h_{1}: C^{1}\left(\bar{B}, \mathbb{R}^{2}\right) \rightarrow \mathbb{R}, \quad h_{1}\left[u_{1}, u_{2}\right]:=u_{1}(0)+u_{2}(0)$;

(vi) $h_{2}: C^{1}\left(\bar{B}, \mathbb{R}^{2}\right) \rightarrow \mathbb{R}, \quad h_{1}\left[u_{1}, u_{2}\right]:=\int_{\partial B} u_{1}^{2}\left(1-\left|\nabla u_{2}\right|^{2}\right) \mathrm{d} \sigma ;$

(vii) $\zeta_{1} \equiv \zeta_{2} \equiv 1$.

Furthermore, it is straightforward to check that all the structural assumptions (I)-to-(VI) listed at the beginning of Section 3 are satisfied (for every $\alpha \in(0,1)$ ). We now aim to show that, in this case, also assumptions (a)-to-(c) in statement of Theorem 3.3 are fulfilled.

Assumption (a). To begin with, we consider the finite sequence

$$
\varrho=\left\{\rho_{1}, \rho_{2}\right\}, \quad \text { where } \rho_{1}=\rho_{2}=\sqrt{\frac{\pi}{6}} .
$$

Clearly, the function $f_{1}$ is continuous and non-negative on $\bar{B} \times I(\varrho) \times R(\varrho)$ (see (3.7) for the definition of $I(\varrho)$ and $R(\varrho)$ ); moreover, since $\rho_{1}, \rho_{2} \leq 4$ and since, by Cauchy-Schwarz inequality, we have (remind the definition of $|\cdot|$ in $(\underline{3.5})$ )

$$
\left|\left\langle\mathbf{w}_{1}, \mathbf{w}_{2}\right\rangle\right| \leq 3\left|\mathbf{w}_{1}\right| \cdot\left|\mathbf{w}_{2}\right| \leq \frac{\pi}{2} \quad \text { for any } \mathbf{w}=\left(\mathbf{w}_{1}, \mathbf{w}_{2}\right) \in R(\varrho),
$$

we easily deduce that also $f_{2}$ is (continuous and) non-negative on $\bar{B} \times I(\varrho) \times R(\varrho)$.

As for the operators $h_{1}, h_{2}$, it is immediate to check that they are (continuous and) nonnegative when restricted to the cone $P(\varrho)$ (note that, if $\mathbf{u} \in P(\varrho)$, we have $\left|\nabla u_{2}\right| \leq \rho_{2}<1$ ); 
furthermore, since $\mathbf{u}=\left(u_{1}, u_{2}\right) \in P(\varrho)$ implies that $0 \leq u_{1}, u_{2} \leq \sqrt{\pi / 6}$, we have

$$
h_{1}[\mathbf{u}]=h_{1}\left[u_{1}, u_{2}\right] \leq 2 \sqrt{\frac{\pi}{6}} \quad \text { and } \quad h_{2}[\mathbf{u}]=h_{2}\left[u_{1}, u_{2}\right] \leq \frac{\pi}{6}|\partial B|=\frac{2 \pi^{2}}{3} .
$$

Thus, $h_{1}, h_{2}$ are bounded on $P(\varrho)$, and this proves that assumption (a) is fulfilled.

Assumption (b). First of all we observe that, by definition, one has

$$
f_{1}(x, \mathbf{z}, \mathbf{w}) \geq e^{z_{1}} \quad \text { for every }(x, \mathbf{z}, \mathbf{w}) \in \bar{B} \times \mathbb{R}^{2} \times \mathbb{R}^{6}
$$

as a consequence, given any $\delta>0$, it is possible to find a small $\rho_{0}=\rho_{0}(\delta) \in(0, \sqrt{\pi / 6})$ such that (here, $I_{0}=\left[0, \rho_{0}\right] \times\left[0, \rho_{0}\right]$ and $R_{0}:=R_{\rho_{0}} \times R_{\rho_{0}}$, see (3.7))

$$
f_{1}(x, \mathbf{z}, \mathbf{w}) \geq e^{z_{1}} \geq \delta z_{1} \quad \text { for every }(x, \mathbf{z}, \mathbf{w}) \in \bar{B} \times I_{0} \times R_{0} .
$$

This proves that $f_{1}$ satisfies (3.9), and thus assumption (b) is fulfilled (with $k_{0}=1$ ).

Assumption (c). We begin by explicitly computing the quantities appearing in (3.10). On the one hand, by the very definition of $f_{1}, f_{2}$ we have

$$
M_{1}=\max _{\bar{B} \times I(\varrho) \times R(\varrho)} f_{1}=e^{\sqrt{\pi / 6}}\left(1+\frac{\pi}{6}\right) \quad \text { and } \quad M_{2}=\max _{\bar{B} \times I(\varrho) \times R(\varrho)} f_{2}=16 .
$$

On the other hand, on account of (4.3), we have (notice that the constant function defined on $\bar{B}$ by $\mathbf{u}:=(\sqrt{\pi / 6}, 0)$ certainly belongs to $P(\varrho))$

$$
H_{1}=\sup _{\mathbf{u} \in P(\varrho)} h_{1}[\mathbf{u}]=2 \sqrt{\frac{\pi}{6}} \quad \text { and } \quad H_{2}=\sup _{\mathbf{u} \in P(\varrho)} h_{2}[\mathbf{u}]=\frac{2 \pi^{2}}{3} .
$$

We now observe that, since $\mathcal{L}_{1}=\mathcal{L}_{2}=-\Delta$ (and taking into account the very definition of Green operator, see (2.9) $)$, one obviously has

$$
\mathcal{G}_{1}(\hat{1})=\mathcal{G}_{\mathcal{L}_{1}}(\hat{1})=\mathcal{G}_{(-\Delta)}(\hat{1}) \quad \text { and } \quad \mathcal{G}_{2}(\hat{1})=\mathcal{G}_{\mathcal{L}_{2}}(\hat{1})=\mathcal{G}_{(-\Delta)}(\hat{1}),
$$

where $\mathcal{G}_{(-\Delta)}(\hat{1})$ is the unique solution of

$$
\left\{\begin{array}{l}
-\Delta u=1 \quad \text { in } B \\
\left.u\right|_{\partial B}=0
\end{array}\right.
$$

As a consequence, since a direct computation gives $\mathcal{G}_{(-\Delta)}(\hat{1})=\frac{1}{2}\left(1-\|x\|^{2}\right)$, we get

$$
\left\|\mathcal{G}_{1}(\hat{1})\right\|_{\infty}=\left\|\mathcal{G}_{2}(\hat{1})\right\|_{\infty}=\frac{1}{2} .
$$

Analogously, since $\zeta_{1} \equiv \zeta_{2} \equiv 1$ (and again since $\mathcal{L}_{1}=\mathcal{L}_{2}=-\Delta$ ), from (3.3) we deduce that $\gamma_{1}=\gamma_{2}=\hat{\gamma}$, where $\hat{\gamma}$ is the unique solution of

$$
\left\{\begin{array}{l}
\Delta u=0 \quad \text { in } B \\
\left.u\right|_{\partial B}=1
\end{array}\right.
$$


As a consequence, since $\hat{\gamma} \equiv 1$ clearly solves the above problem, we get

$$
\left\|\gamma_{1}\right\|_{\infty}=\left\|\gamma_{2}\right\|_{\infty}=1
$$

Finally, according to (3.11), we turn to provide an explicit estimate for

$$
\sup _{x \in B} \int_{B}\left|\partial_{x_{l}} g_{(-\Delta)}(y ; x)\right| \mathrm{d} y \quad(\text { with } l=1,2,3),
$$

where $g_{(-\Delta)}$ is the Green function for $(-\Delta)$ (and related to $B$ ). To this end, we make crucial use of the explicit expression of $g_{(-\Delta)}$ (see, e.g., [11, Section 2.2.4-(c)])):

$$
g_{(-\Delta)}(y ; x)=\frac{1}{4 \pi}\left(\|x-y\|^{-1}-\left(1+\|x\|^{2}\|y\|^{2}-2\langle x, y\rangle\right)^{-1 / 2}\right)
$$

where $\|\cdot\|$ is the usual Euclidean norm in $\mathbb{R}^{3}$. Starting from (4.8), a direct yet tedious computation shows that (for every $x, y \in B$ with $x \neq y$ )

$$
\left|\partial_{x_{l}} g_{(-\Delta)}(y ; x)\right| \leq \frac{1}{2 \pi\|x-y\|^{2}}
$$

as a consequence, for every $x \in B$ we have

$$
\begin{gathered}
\int_{B}\left|\partial_{x_{l}} g_{(-\Delta)}(y ; x)\right| \mathrm{d} y \leq \frac{1}{2 \pi} \int_{B}\|x-y\|^{-2} \mathrm{~d} y \leq \frac{1}{2 \pi} \int_{\{\|x-y\|<2\}}\|x-y\|^{-2} \mathrm{~d} y \\
=\frac{1}{2 \pi} \int_{\{\|y\|<2\}}\|y\|^{-2} \mathrm{~d} y=\frac{1}{2 \pi}|\partial B| \int_{0}^{2} \mathrm{~d} \rho=4 .
\end{gathered}
$$

Thus, taking into account that $\mathcal{L}_{1}=\mathcal{L}_{2}=-\Delta$, we obtain

$$
\mathcal{G}_{1, l}=\mathcal{G}_{2, l}=\sup _{x \in B} \int_{B}\left|\partial_{x_{l}} g_{(-\Delta)}(y ; x)\right| \mathrm{d} y \leq 4, \quad \text { for every } l=1,2,3 .
$$

By gathering together (4.2), (4.4), (4.5), (4.6), (4.7) and (4.9), we are finally entitled to apply Theorem 3.3: for any $\lambda_{1}>0$ and any $\lambda_{2}, \eta_{1}, \eta_{2} \geq 0$ satisfying$$
\left.\frac{\lambda_{1}}{2} e^{\sqrt{\pi / 6}}\left(1+\frac{\pi}{6}\right)+2 \eta_{1} \sqrt{\frac{\pi}{6}} \leq \sqrt{\frac{\pi}{6}} \quad \text { (see assumption }(\mathrm{c})_{2}\right),
$$$$
\text { (*) } \left.\quad \lambda_{2}+\frac{2 \pi^{2}}{3} \eta_{2} \leq \sqrt{\frac{\pi}{6}} ; \quad \text { (see assumption }(\mathrm{c})_{2}\right),
$$

$$
\left.\max \left\{4 \lambda_{1} e^{\sqrt{\pi / 6}}\left(1+\frac{\pi}{6}\right), 64 \lambda_{2}\right\} \leq \sqrt{\frac{\pi}{6}}, \quad \text { (see assumption }(\mathrm{c})_{3}\right),
$$

there exists at least one solution $\mathbf{u}=\left(u_{1}, u_{2}\right) \in C^{1}\left(\bar{B}, \mathbb{R}^{2}\right)$ of (4.1) such that

$$
\left\|u_{1}\right\|_{\infty},\left\|u_{2}\right\|_{\infty} \leq \sqrt{\frac{\pi}{6}} \quad \text { and } \quad\|\mathbf{u}\|_{C^{1}\left(\bar{B}, \mathbb{R}^{2}\right)} \geq \rho_{0} .
$$


Here, $\rho_{0}=\rho_{0}(\delta)>0$ is as in assumption (b) and $\delta>0$ is such that $\mu_{1} \leq \delta \lambda_{1}$ (see assumption (c) ${ }_{1}$ and remind that $\mu_{1}>0$ denotes the inverse of the spectral radius of $\mathcal{L}_{1}=-\Delta$, see (3.4)). It should be noticed that, since (3.9) holds for any given $\delta>0$ (by accordingly choosing $\left.\rho_{0}=\rho_{0}(\delta)>0\right)$, there is no need to have an explicit knowledge of $\mu_{1}$.

Example 4.2. On Euclidean space $\mathbb{R}^{3}$, let us consider the following BVP

$$
\begin{cases}-\Delta u_{1}=\lambda_{1} u_{1}^{2}\left(1-e^{-\left|\nabla u_{2}\right|}\right) & \text { in } B \\ -\Delta u_{2}=\lambda_{2} \sin \left(u_{2}\right)\left(u_{1}^{3}+\left|\left\langle\nabla u_{1}, \nabla u_{2}\right\rangle\right|\right) & \text { in } B \\ \left.u_{1}\right|_{\partial B}=\eta_{1} \int_{B} u_{2}^{2} \mathrm{~d} x, & \\ \left.u_{2}\right|_{\partial B}=\eta_{2} \max _{\partial B} u_{1}, & \end{cases}
$$

where $B$ is the Euclidean ball with centre 0 and radius 1 and we adopt the same notation of Example 4.1.

Obviously, this problem takes the form (3.1) with

(i) $\mathcal{O}:=B$;

(ii) $\mathcal{L}_{1}=\mathcal{L}_{2}=-\Delta$;

(iii) $f_{1}: \bar{B} \times \mathbb{R}^{2} \times \mathbb{R}^{6} \rightarrow \mathbb{R}, \quad f_{1}(x, \mathbf{z}, \mathbf{w})=z_{1}^{2}\left(1-e^{\left|\mathbf{w}_{2}\right|}\right)$;

(iv) $f_{2}: \bar{B} \times \mathbb{R}^{2} \times \mathbb{R}^{6} \rightarrow \mathbb{R}, \quad f_{2}(x, \mathbf{z}, \mathbf{w})=\sin \left(z_{2}\right)\left(z_{1}^{3}+\left|\left\langle\mathbf{w}_{1}, \mathbf{w}_{2}\right\rangle\right|\right)$;

(v) $h_{1}: C^{1}\left(\bar{B}, \mathbb{R}^{2}\right) \rightarrow \mathbb{R}, \quad h_{1}\left[u_{1}, u_{2}\right]:=\int_{B} u_{2}^{2} \mathrm{~d} x$;

(vi) $h_{2}: C^{1}\left(\bar{B}, \mathbb{R}^{2}\right) \rightarrow \mathbb{R}, \quad h_{1}\left[u_{1}, u_{2}\right]:=\max _{\partial B} u_{1}$;

(vii) $\zeta_{1} \equiv \zeta_{2} \equiv 1$.

Furthermore, it is straightforward to check that all the structural assumptions (I)-to-(VI) listed at the beginning of Section 3 are satisfied (for every $\alpha \in(0,1)$ ). We now aim to show that, in this case, assumptions (a)-to-(c) in statement of Theorem 3.5 are fulfilled.

Assumption (a). To begin with, we consider the finite sequence

$$
\varrho=\left\{\rho_{1}, \rho_{2}\right\}, \quad \text { where } \rho_{1}=\rho_{2}=1 \text {. }
$$

Clearly, the function $f_{1}$ is continuous and non-negative on $\bar{B} \times I(\varrho) \times R(\varrho)$; moreover, for every $(x, \mathbf{z}, \mathbf{w}) \in \bar{B} \times I(\varrho) \times R(\varrho)$ one has (notice that, if $z \in I(\varrho)$, then $0 \leq z_{1} \leq 1$ )

$$
0 \leq f_{1}(x, \mathbf{z}, \mathbf{w})=z_{1} \cdot\left(z_{1}\left(1-e^{-\left|\mathbf{w}_{2}\right|}\right)\right) \leq u_{1}
$$

Thus, $f_{1}$ fulfills assumption (a) (with $\tau_{1}=1$ ). 
As regards $f_{2}$, we obviously have that also this function is continuous and non-negative on $\bar{B} \times I(\varrho) \times R(\varrho)$; moreover, since $0 \leq \sin (t) \leq t$ for every $0 \leq t \leq 1$, we have

$$
0 \leq f_{2}(x, \mathbf{z}, \mathbf{w}) \leq z_{2}\left(1+\left|\left\langle\mathbf{w}_{1}, \mathbf{w}_{2}\right\rangle\right|\right)
$$

(by Cauchy-Schwarz inequality, see Example 4.1)

$$
\begin{aligned}
& \leq z_{2}\left(1+3\left|\mathbf{w}_{1}\right| \cdot\left|\mathbf{w}_{2}\right|\right) \\
& \text { (since } \left.w=\left(\mathbf{w}_{1}, \mathbf{w}_{2}\right) \in R(\varrho) \text { implies that }\left|\mathbf{w}_{1}\right|,\left|\mathbf{w}_{2}\right| \leq 1\right) \\
& \leq 4 z_{2} \quad(\text { for every }(x, z, w) \in \bar{B} \times I(\varrho) \times R(\varrho)) .
\end{aligned}
$$

As a consequence, also $f_{2}$ satisfies assumption (a) (with $\tau_{2}=4$ ).

Assumption (b). First of all, it is very easy to check that both $h_{1}$ and $h_{2}$ are continuous and non-negative when restricted to the cone $P(\varrho) \subseteq C^{1}(\bar{B}, \mathbb{R})$; moreover, since the condition $\mathbf{u}=\left(u_{1}, u_{2}\right) \in P(\varrho)$ implies that $0 \leq u_{1}, u_{2} \leq 1$, we get

$$
h_{1}[\mathbf{u}]=h_{1}\left[u_{1}, u_{2}\right] \leq \int_{B} u_{2} \mathrm{~d} x \leq\left(\max _{\bar{B}} u_{2}\right) \cdot\left|B_{1}\right| \leq \frac{4 \pi}{3}\|\mathbf{u}\|_{\infty},
$$

and this proves that $h_{1}$ fulfills assumption (b) (with $\xi_{1}=(4 \pi) / 3$ ).

Finally, by exploiting the very definition of $\|\cdot\|_{\infty}$, we have

$$
h_{2}[\mathbf{u}]=h_{2}\left[u_{1}, u_{2}\right]=\max _{\partial B} u_{1} \leq\|\mathbf{u}\|_{\infty},
$$

and thus also $h_{2}$ satisfies assumption (b) (with $\xi_{2}=1$ ).

Assumption (c). By making use of all the computations already carried out in the previous Example 4.1. we know that (see, precisely, (4.6) and (4.7))

(i) $\left\|\mathcal{G}_{1}(\hat{1})\right\|_{\infty}=\left\|\mathcal{G}_{2}(\hat{1})\right\|_{\infty}=1 / 2$;

(ii) $\left\|\gamma_{1}\right\|_{\infty}=\left\|\gamma_{2}\right\|_{\infty}=1$.

As a consequence, by gathering together (4.11), (4.12), (4.13), (4.14), (4.15) and the above (i)-(ii), we are entitled to apply Theorem 3.5: for any $\lambda_{1}, \lambda_{2}, \eta_{1}, \eta_{2} \geq 0$ satisfying

$$
\frac{\lambda_{1}}{2}+\frac{4 \pi}{3} \eta_{1}<1 \quad \text { and } \quad 2 \lambda_{2}+\eta_{2}<1
$$

the BVP (4.10) possesses only the zero solution (notice that $\mathbf{u} \equiv 0$ trivially solves (4.10)).

\section{ReFerenCes}

[1] C.O. Alves and A. Moussaoui, Existence of solutions for a class of singular elliptic systems with convection term, Asymptot. Anal., 90 (2014), 237-248.

[2] H. Amann, Fixed point equations and nonlinear eigenvalue problems in ordered Banach spaces, SIAM. Rev., 18 (1976), 620-709. 
[3] H. Amann and M.G. Crandall, On some existence theorems for semi-linear elliptic equations, Indiana Univ. Math. J., 27 (1978), no. 5, 779-790.

[4] H. Brézis and R.E.L. Turner, On a class of superlinear elliptic problems, Comm. Partial Differential Equations, 2 (1977) 601-614.

[5] F. Cianciaruso, G. Infante and P. Pietramala, Solutions of perturbed Hammerstein integral equations with applications, Nonlinear Anal. Real World Appl., 33 (2017), 317-347.

[6] F. Cianciaruso, G. Infante and P. Pietramala, Non-zero radial solutions for elliptic systems with coupled functional BCs in exterior domains, Proc. Edinb. Math. Soc., 62 (2019), 747-769.

[7] D. O'Regan and R. Precup, Theorems of Leray-Schauder type and applications, Series in Mathematical Analysis and Applications, 3. Gordon and Breach Science Publishers, Amsterdam, 2001.

[8] D.G. de Figueiredo, Nonvariational Semilinear Elliptic Systems. In: Lavor C., Gomes F. (eds) Advances in Mathematics and Applications. Springer, Cham, (2018), 131-151.

[9] D.G. De Figueiredo, M. Girardi and M. Matzeu, Semilinear elliptic equations with dependence on the gradient via mountain-pass techniques, Differential Integral Equations, 17 (2004), 119-126.

[10] D.G. De Figueiredo and J. Yang, A priori bounds for positive solutions of a non-variational elliptic system, Comm. Partial Differential Equations, 26 (2001), 2305-2321.

[11] L.C. Evans, Partial differential equations, American Mathematical Society, Providence, RI, (2010).

[12] D. Gilbarg and N.S. Trudinger, Elliptic Partial Differential Equations of Second Order, SpringerVerlag, Berlin, (2011).

[13] M. Girardi and M. Matzeu, Positive and negative solutions of a quasi-linear elliptic equation by a mountain pass method and truncature techniques, Nonlinear Anal., 59 (2004), 199-210.

[14] C. S. Goodrich, New Harnack inequalities and existence theorems for radially symmetric solutions of elliptic PDEs with sign changing or vanishing Green's function, J. Differential Equations, 264 (2018), 236-262.

[15] C. S. Goodrich, Radially symmetric solutions of elliptic PDEs with uniformly negative weight, Ann. Mat. Pura Appl., 197 (2018), 1585-1611.

[16] D. Guo and V. Lakshmikantham, Nonlinear problems in abstract cones, Academic Press, Boston, (1988).

[17] G. Infante, Nonzero positive solutions of a multi-parameter elliptic system with functional BCs, Topol. Methods Nonlinear Anal., 52 (2018), 665-675.

[18] G. Infante, Nonzero positive solutions of nonlocal elliptic systems with functional BCs, J. Elliptic Parabol. Equ., 5 (2019), 493-505.

[19] G. Infante, Positive and increasing solutions of perturbed Hammerstein integral equations with derivative dependence, Discrete Contin. Dyn. Syst. Ser. B, 25 (2020), 691-699.

[20] G. Infante, Positive solutions of systems of perturbed Hammerstein integral equations with arbitrary order dependence, Philos. Trans. Roy. Soc. A, to appear.

[21] S. Kim and G. Sakellaris, Green's function for second order elliptic equations with singular lower order coefficients, Comm. Partial Differential Equations, 44 (2019), 228-270.

[22] J. Mawhin and K. Schmitt, Upper and lower solutions and semilinear second order elliptic equations with nonlinear boundary conditions, Proc. Roy. Soc. Edinburgh Sect. A, 97 (1984), 199-207. 
[23] J. Mawhin and K. Schmitt, Corrigendum to "Upper and lower solutions and semilinear second order elliptic equations with nonlinear boundary conditions", Proc. Roy. Soc. Edinburgh Sect. A, 100 (1985), 361.

[24] C. V. Pao and Y. M. Wang, Nonlinear fourth-order elliptic equations with nonlocal boundary conditions, J. Math. Anal. Appl., 372 (2010), 351-365.

[25] S. I. Pokhozhaev, On equations of the form $-\Delta u=f(x, u, D u)$, Math. USSR, Sb., 41 (1982), 269-280.

[26] D. Ruiz and A. Suárez, Existence and uniqueness of positive solution of a logistic equation with nonlinear gradient term, Proc. Roy. Soc. Edinburgh Sect. A, 137 (2007), 555-566.

[27] X.J. Wang and Y.B. Deng, Existence of multiple solutions to nonlinear elliptic equations of nondivergence form, J. Math. Anal. Appl., 189 (1995), 617-630.

[28] J. R. L. Webb, Existence of positive solutions for a thermostat model, Nonlinear Anal. Real World Appl., 13 (2012), 923-938.

[29] Z.Q. Yan, A note on the solvability in $W^{2, p}(\Omega)$ for the equation $-\Delta u=f(x, u, D u)$, Nonlinear Anal., 24 (1995), 1413-1416.

Stefano Biagi, Dipartimento di Dipartimento di Ingegneria Industriale e Scienze Matematiche, Università Politecnica delle Marche, Via Brecce Bianche, 60131 Ancona, Italy

E-mail address: biagi@dipmat.univpm.it

Alessandro Calamai, Dipartimento di Ingegneria Civile, Edile e Architettura, Università Politecnica delle Marche, Via Brecce Bianche, 60131 Ancona, Italy

E-mail address: calamai@dipmat.univpm.it

Gennaro Infante, Dipartimento di Matematica e Informatica, Università della Calabria, 87036 Arcavacata di Rende, Cosenza, Italy

E-mail address: gennaro.infante@unical.it 